\title{
Evaluation Trees for Proposition Algebra
}

\author{
Jan A. Bergstra \& Alban Ponse \\ Section Theory of Computer Science, Informatics Institute \\ Faculty of Science, University of Amsterdam \\ https://staff.science.uva.nl/\{j.a.bergstra/,a.ponse/\}
}

\begin{abstract}
Proposition algebra is based on Hoare's conditional connective, which is a ternary connective comparable to if-then-else and used in the setting of propositional logic. Conditional statements are provided with a simple semantics that is based on evaluation trees and that characterizes so-called free valuation congruence: two conditional statements are free valuation congruent if, and only if, they have equal evaluation trees. Free valuation congruence is axiomatized by the four basic equational axioms of proposition algebra that define the conditional connective. Valuation congruences that identify more conditional statements than free valuation congruence are repetition-proof, contractive, memorizing, and static valuation congruence. Each of these valuation congruences is characterized using a transformation on evaluation trees: two conditional statements are C-valuation congruent if, and only if, their C-transformed evaluation trees are equal. These transformations are simple and natural, and only for static valuation congruence a slightly more complex transformation is used. Also, each of these valuation congruences is axiomatized in proposition algebra. A spin-off of our approach can be called "normalization functions for proposition algebra": for each valuation congruence $\mathrm{C}$ considered, two conditional statements are $\mathrm{C}$-valuation congruent if, and only if, the C-normalization function returns equal images.
\end{abstract}

Keywords: Conditional composition, evaluation tree, proposition algebra, short-circuit evaluation, short-circuit logic, side effect

\section{Contents}

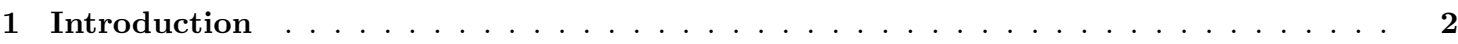

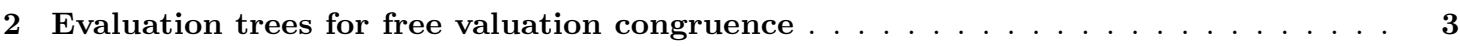

3 Evaluation trees for repetition-proof valuation congruence . . . . . . . . . . . 8

4 Evaluation trees for contractive valuation congruence . . . . . . . . . . . . 15

5 Evaluation trees for memorizing valuation congruence . . . . . . . . . . . . 20

6 Evaluation trees for static valuation congruence . . . . . . . . . . . . . . 26

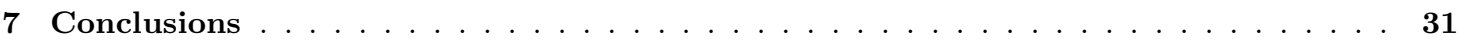




$$
\begin{aligned}
x \triangleleft \mathrm{T} \triangleright y & =x \\
x \triangleleft \mathrm{F} \triangleright y & =y \\
\mathrm{~T} \triangleleft x \triangleright \mathrm{F} & =x \\
x \triangleleft(y \triangleleft z \triangleright u) \triangleright v & =(x \triangleleft y \triangleright v) \triangleleft z \triangleright(x \triangleleft u \triangleright v)
\end{aligned}
$$

Table 1: The set CP of equational axioms for free valuation congruence

\section{Introduction}

In 1985, Hoare's paper A couple of novelties in the propositional calculus [1] was published. In this paper the ternary connective $\_\triangleleft_{-} \triangleright_{-}$is introduced as the conditional 1 A more common expression for a conditional statement

$$
P \triangleleft Q \triangleright R
$$

is

$$
\text { if } Q \text { then } P \text { else } R \text {, }
$$

but in order to reason systematically with conditional statements, a notation such as $P \triangleleft Q \triangleright R$ is preferable. In a conditional statement $P \triangleleft Q \triangleright R$, first $Q$ is evaluated, and depending on that evaluation result, either $P$ or $R$ is evaluated (and the other is not) and determines the evaluation value. This evaluation strategy is a form of short-circuit evaluation 2 In [1], Hoare proves that propositional logic is characterized by eleven equational axioms, some of which employ constants $\mathrm{T}$ and $\mathrm{F}$ for the truth values true and false.

In 2011, we introduced Proposition Algebra in 4 as a general approach to the study of the conditional: we defined several valuation congruences and provided equational axiomatizations of these congruences. The most basic and least identifying valuation congruence is free valuation congruence, which is axiomatized by the axioms in Table 1. These axioms stem from 11 and define the conditional as a primitive connective. We use the name CP (for Conditional Propositions) for this set of axioms. Interpreting a conditional statement as an if-then-else expression, axioms (CP1)-(CP3) are natural, and axiom (CP4) (distributivity) can be clarified by case analysis: if $z$ evaluates to true and $y$ as well, then $x$ determines the result of evaluation; if $z$ evaluates to true and $y$ evaluates to false, then $v$ determines the result of evaluation, and so on and so forth. A simple example, taken from [4, is the conditional statement that a pedestrian evaluates before crossing a road with two-way traffic driving on the right:

$$
(\text { look-left-and-check } \triangleleft \text { look-right-and-check } \triangleright \mathrm{F}) \triangleleft \text { look-left-and-check } \triangleright \mathrm{F} \text {. }
$$

This statement requires one, or two, or three atomic evaluations and cannot be simplified to one that requires less ${ }^{3}$

In $\$ 2$ we characterize free valuation congruence with help of evaluation trees: given a conditional statement, its evaluation tree directly represents all its evaluations (in the way a truth

\footnotetext{
${ }^{1}$ To be distinguished from Hoare's conditional introduced in his 1985 book on CSP [10] and in his well-known 1987 paper Laws of Programming 9 for expressions $P \triangleleft b \triangleright Q$ with $P$ and $Q$ denoting programs and $b$ a Boolean expression.

${ }^{2}$ Short-circuit evaluation denotes the semantics of binary propositional connectives in which the second argument is evaluated only if the first argument does not suffice to determine the value of the expression.

${ }^{3}$ Note that look-left-and-check $\triangleleft($ look-right-and-check $\triangleleft$ look-left-and-check $\triangleright \mathrm{F}) \triangleright \mathrm{F}$ prescribes by axioms $\mathrm{CP} 4$ and (CP2) the same evaluation.
} 
table does in the case of propositional logic). Two conditional statements are equivalent with respect to free valuation congruence if their evaluation trees are equal. Evaluation trees are simple binary trees, proposed by Daan Staudt in [14] (that appeared in 2012). Free valuation congruence identifies less than the equivalence defined by Hoare's axioms in 11. For example, the atomic proposition $a$ and the conditional statement $\mathrm{T} \triangleleft a \triangleright a$ are not equivalent with respect to free valuation congruence, although they are equivalent with respect to static valuation congruence, which is the valuation congruence that characterizes propositional logic.

In 93 we consider repetition-proof valuation congruence, a valuation congruence that identifies more than free and less than static valuation congruence. Repetition-proof valuation congruence is axiomatized by $\mathrm{CP}$ extended with two (schematic) axioms, one of which reads

$$
x \triangleleft a \triangleright(y \triangleleft a \triangleright z)=x \triangleleft a \triangleright(z \triangleleft a \triangleright z)
$$

for any atomic proposition $a$, and thus expresses that if $a$ evaluates to false, a consecutive evaluation of $a$ also evaluates to false, so the conditional statement at the $y$-position will not be evaluated and can be replaced by any other. As an example,

$$
\mathrm{T} \triangleleft a \triangleright a=\mathrm{T} \triangleleft a \triangleright(\mathrm{T} \triangleleft a \triangleright \mathrm{F})=\mathrm{T} \triangleleft a \triangleright(\mathrm{F} \triangleleft a \triangleright \mathrm{F}),
$$

and the left-hand and right-hand conditional statements are equivalent with respect to repetitionproof valuation congruence, but not with respect to free valuation congruence. We characterize repetition-proof valuation congruence by defining a transformation on evaluation trees that yields repetition-proof evaluation trees: two conditional statements are equivalent with respect to repetition-proof valuation congruence if, and only if, they have equal repetition-proof evaluation trees. Although this transformation on evaluation trees is simple and natural, our proof of the mentioned characterization - which is phrased as a completeness result - is non-trivial and we could not find a proof that is essentially simpler.

Valuation congruences that identify more conditional statements than repetition-proof valuation congruence are contractive, memorizing, and static valuation congruence, and these are all defined and axiomatized in [4]. In $\S \Phi 4$, each of these valuation congruences is characterized using a transformation on evaluation trees: two conditional statements are C-valuation congruent if, and only if, their C-transformed evaluation trees are equal. These transformations are simple and natural, and only for static valuation congruence we use a slightly more complex transformation.

In 97 we discuss the general structure of the proofs of the axiomatization results in $\S \$ 3$, each of which is based on a normalization function for conditional statements. Then we end the paper with a brief digression on short-circuit logic, an example on the use of repetition-proof valuation congruence, and some remarks about side effects.

A shortened version of this paper, not covering the material in $\$ \$ 4$ 6 6 , was published as [5]. However, we simplified the proof of Lemma 3.15 in [5] (Lemma 3.14 in this version).

\section{Evaluation trees for free valuation congruence}

Consider the signature $\Sigma_{\mathrm{CP}}(A)=\left\{\_\triangleleft_{-} \triangleright_{-}, \mathrm{T}, \mathrm{F}, a \mid a \in A\right\}$ with constants $\mathrm{T}$ and $\mathrm{F}$ for the truth values true and false, respectively, and constants $a$ for atomic propositions, further called atoms, from some countable set $A$ containing at least two atoms. We write

$$
C_{A}
$$

for the set of closed terms, or conditional statements, over the signature $\Sigma_{\mathrm{CP}}(A)$. Given a conditional statement $P \triangleleft Q \triangleright R$, we refer to $Q$ as its central condition. 
We define the dual $P^{d}$ of $P \in C_{A}$ as follows:

$$
\begin{array}{rlrl}
\mathrm{T}^{d} & =\mathrm{F}, \\
\mathrm{F}^{d} & =\mathrm{T}, & a^{d} & =a \quad(\text { for } a \in A) \\
& (P \triangleleft Q \triangleright R)^{d} & =R^{d} \triangleleft Q^{d} \triangleright P^{d} .
\end{array}
$$

Observe that CP is a self-dual axiomatization: when defining $x^{d}=x$ for each variable $x$, the dual of each axiom is also in $\mathrm{CP}$, and hence

$$
\mathrm{CP} \vdash P=Q \quad \Longleftrightarrow \quad \mathrm{CP} \vdash P^{d}=Q^{d} .
$$

A natural view on conditional statements in $C_{A}$ involves short-circuit evaluation, similar to how we consider the evaluation of an "if $y$ then $x$ else $z$ " expression. The following definition is taken from [14.

Definition 2.1. The set $\mathcal{T}_{A}$ of evaluation trees over $A$ with leaves in $\{\mathrm{T}, \mathrm{F}\}$ is defined inductively by

$$
\begin{aligned}
\mathrm{T} & \in \mathcal{T}_{A}, \\
\mathrm{~F} & \in \mathcal{T}_{A}, \\
(X \unlhd a \unrhd Y) & \in \mathcal{T}_{A} \text { for any } X, Y \in \mathcal{T}_{A} \text { and } a \in A .
\end{aligned}
$$

The function $\_\unlhd a \unrhd_{-}$is called post-conditional composition over a. In the evaluation tree $X \unlhd a \unrhd Y$, the root is represented by $a$, the left branch by $X$ and the right branch by $Y$.

We refer to trees in $\mathcal{T}_{A}$ as evaluation trees, or trees for short. Post-conditional composition and its notation stem from [2]. Evaluation trees play a crucial role in the main results of [14. In order to define our "evaluation tree semantics", we first define an auxiliary function on trees.

Definition 2.2 (Leaf replacement). Given evaluation trees $Y, Z \in \mathcal{T}_{A}$, the leaf replacement function $[T \mapsto Y, F \mapsto Z]: \mathcal{T}_{A} \rightarrow \mathcal{T}_{A}$ for which postfix notation

$$
X[\mathrm{~T} \mapsto Y, \mathrm{~F} \mapsto Z]
$$

is adopted, is defined as follows, where $a \in A$ :

$$
\begin{aligned}
& \mathrm{T}[\mathrm{T} \mapsto Y, \mathrm{~F} \mapsto Z]=Y, \\
& \mathrm{~F}[\mathrm{~T} \mapsto Y, \mathrm{~F} \mapsto Z]=Z, \\
& \left(X_{1} \unlhd a \unrhd X_{2}\right)[\mathrm{T} \mapsto Y, \mathrm{~F} \mapsto Z]=X_{1}[\mathrm{~T} \mapsto Y, \mathrm{~F} \mapsto Z] \unlhd a \unrhd X_{2}[\mathrm{~T} \mapsto Y, \mathrm{~F} \mapsto Z] .
\end{aligned}
$$

We note that the order in which the replacements of leaves of $X$ is listed is irrelevant and adopt the convention of not listing identities inside the brackets, e.g., $X[\mathrm{~F} \mapsto Z]=X[\mathrm{~T} \mapsto \mathrm{T}, \mathrm{F} \mapsto Z]$. Furthermore, repeated replacements satisfy the following equation:

$$
\begin{aligned}
\left(X\left[\mathrm{~T} \mapsto Y_{1}, \mathrm{~F} \mapsto Z_{1}\right]\right)\left[\mathrm{T} \mapsto Y_{2}, \mathrm{~F} \mapsto Z_{2}\right] \\
=X\left[\mathrm{~T} \mapsto Y_{1}\left[\mathrm{~T} \mapsto Y_{2}, \mathrm{~F} \mapsto Z_{2}\right], \mathrm{F} \mapsto Z_{1}\left[\mathrm{~T} \mapsto Y_{2}, \mathrm{~F} \mapsto Z_{2}\right]\right] .
\end{aligned}
$$

We now have the terminology and notation to define the interpretation of conditional statements in $C_{A}$ as evaluation trees by a function se (abbreviating short-circuit evaluation). 
Definition 2.3. The short-circuit evaluation function se $C_{A} \rightarrow \mathcal{T}_{A}$ is defined as follows, where $a \in A$ :

$$
\begin{aligned}
s e(\mathrm{~T}) & =\mathrm{T}, \\
s e(\mathrm{~F}) & =\mathrm{F}, \\
s e(a) & =\mathrm{T} \unlhd a \unrhd \mathrm{F}, \\
s e(P \triangleleft Q \triangleright R) & =s e(Q)[\mathrm{T} \mapsto s e(P), \mathrm{F} \mapsto s e(R)] .
\end{aligned}
$$

Example 2.4. The conditional statement $a \triangleleft(\mathrm{F} \triangleleft a \triangleright T) \triangleright \mathrm{F}$ yields the following evaluation tree:

$$
\begin{aligned}
s e(a \triangleleft(\mathrm{F} \triangleleft a \triangleright \mathrm{T}) \triangleright \mathrm{F}) & =s e(\mathrm{~F} \triangleleft a \triangleright \mathrm{T})[\mathrm{T} \mapsto s e(a), \mathrm{F} \mapsto s e(\mathrm{~F})] \\
& =(\mathrm{F} \unlhd a \unrhd \mathrm{T})[\mathrm{T} \mapsto s e(a)] \\
& =\mathrm{F} \unlhd a \unrhd(\mathrm{T} \unlhd a \unrhd \mathrm{F}) .
\end{aligned}
$$

A more pictorial representation of this evaluation tree is the following, where $\unlhd$ yields a left branch and $\unrhd$ a right branch:

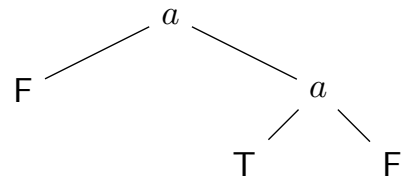

End example.

As we can see from the definition on atoms, evaluation continues in the left branch if an atom evaluates to true and in the right branch if it evaluates to false. We shall often use the constants $\mathrm{T}$ and $\mathrm{F}$ to denote the result of an evaluation (instead of true and false).

Definition 2.5. Let $P \in C_{A}$. An evaluation of $P$ is a pair $(\sigma, B)$ where $\sigma \in(A\{\mathrm{~T}, \mathrm{~F}\})^{*}$ and $B \in\{\mathrm{T}, \mathrm{F}\}$, such that if $\operatorname{se}(P) \in\{\mathrm{T}, \mathrm{F}\}$, then $\sigma=\epsilon$ (the empty string) and $B=\operatorname{se}(P)$, and otherwise,

$$
\sigma=a_{1} B_{1} a_{2} B_{2} \cdots a_{n} B_{n}
$$

where $a_{1} a_{2} \cdots a_{n} B$ is a complete path in se $(P)$ and

- for $i<n$, if $a_{i+1}$ is a left child of $a_{i}$ then $B_{i}=\mathrm{T}$, and otherwise $B_{i}=\mathrm{F}$,

- if $B$ is a left child of $a_{n}$ then $B_{n}=\mathrm{T}$, and otherwise $B_{n}=\mathrm{F}$.

We refer to $\sigma$ as the evaluation path and to $B$ as the evaluation result.

So, an evaluation of a conditional statement $P$ is a complete path in $s e(P)$ (from root to leaf) and contains evaluation values for all occurring atoms. For instance, the evaluation tree $\mathrm{F} \unlhd a \unrhd(\mathrm{T} \unlhd a \unrhd \mathrm{F})$ from Example 2.4 encodes the evaluations $(a \mathrm{~T}, \mathrm{~F}),(a \mathrm{~F} a \mathrm{~T}, \mathrm{~T})$, and $(a \mathrm{~F} a \mathrm{~F}, \mathrm{~F})$. As an aside, we note that this particular evaluation tree encodes all possible evaluations of $\neg a$ \&\& $a$, where \&\& is the connective that prescribes short-circuit conjunction (we return to this connective in 977 .

In turn, each evaluation tree gives rise to a unique conditional statement. For Example 2.4 this is $\mathrm{F} \triangleleft a \triangleright(\mathrm{T} \triangleleft a \triangleright \mathrm{F})$ (note the syntactical correspondence).

Definition 2.6. Basic forms over $A$ are defined by the following grammar

$$
t::=\mathrm{T}|\mathrm{F}| t \triangleleft a \triangleright t \quad \text { for } a \in A .
$$

We write $B F_{A}$ for the set of basic forms over $A$. The depth $d(P)$ of $P \in B F_{A}$ is defined by $d(\mathbf{T})=d(\mathrm{~F})=0$ and $d(Q \triangleleft a \triangleright R)=1+\max \{d(Q), d(R)\}$. 
The following lemma's exploit the structure of basic forms and are stepping stones to our first completeness result (Theorem 2.11).

Lemma 2.7. For each $P \in C_{A}$ there exists $Q \in B F_{A}$ such that $\mathrm{CP} \vdash P=Q$.

Proof. First we establish an auxiliary result: if $P, Q, R$ are basic forms, then there is a basic form $S$ such that $\mathrm{CP} \vdash P \triangleleft Q \triangleright R=S$. This follows by structural induction on $Q$.

The lemma's statement follows by structural induction on $P$. The base cases $P \in\{\mathrm{T}, \mathrm{F}, a \mid$ $a \in A\}$ are trivial, and if $P=P_{1} \triangleleft P_{2} \triangleright P_{3}$ there exist by induction basic forms $Q_{i}$ such that $\mathrm{CP} \vdash P_{i}=Q_{i}$, hence $\mathrm{CP} \vdash P_{1} \triangleleft P_{2} \triangleright P_{3}=Q_{1} \triangleleft Q_{2} \triangleright Q_{3}$. Now apply the auxiliary result.

Lemma 2.8. For all basic forms $P$ and $Q$, se $(P)=\operatorname{se}(Q)$ implies $P=Q$.

Proof. By structural induction on $P$. The base cases $P \in\{\mathrm{T}, \mathrm{F}\}$ are trivial. If $P=P_{1} \triangleleft a \triangleright P_{2}$, then $Q \notin\{\mathrm{T}, \mathrm{F}\}$ and $Q \neq Q_{1} \triangleleft b \triangleright Q_{2}$ with $b \neq a$, so $Q=Q_{1} \triangleleft a \triangleright Q_{2}$ and $\operatorname{se}\left(P_{i}\right)=\operatorname{se}\left(Q_{i}\right)$. By induction we find $P_{i}=Q_{i}$, and hence $P=Q$.

Definition 2.9. Free valuation congruence, notation $=_{s e}$, is defined on $C_{A}$ as follows:

$$
P={ }_{s e} Q \Longleftrightarrow \operatorname{se}(P)=\operatorname{se}(Q) .
$$

Lemma 2.10. Free valuation congruence is a congruence relation.

Proof. Assume $P={ }_{s e} P^{\prime}, Q={ }_{s e} Q^{\prime}$, and $R={ }_{s e} R^{\prime}$. Then $s e(P \triangleleft Q \triangleright R)=s e(Q)[\mathrm{T} \mapsto s e(P), \mathrm{F} \mapsto$ $\operatorname{se}(R)]=\operatorname{se}\left(Q^{\prime}\right)\left[\mathrm{T} \mapsto \operatorname{se}\left(P^{\prime}\right), \mathrm{F} \mapsto \operatorname{se}\left(R^{\prime}\right)\right]=\operatorname{se}\left(P^{\prime} \triangleleft Q^{\prime} \triangleright R^{\prime}\right)$, so $P \triangleleft Q \triangleright R=s e P^{\prime} \triangleleft Q^{\prime} \triangleright R^{\prime}$.

Theorem 2.11 (Completeness of CP). For all $P, Q \in C_{A}$,

$$
\mathrm{CP} \vdash P=Q \quad \Longleftrightarrow P==_{s e} Q .
$$

Proof. $\left(\Rightarrow\right.$ By Lemma 2.10, $=_{s e}$ is a congruence relation and it easily follows that all CP-axioms are sound. For example, soundness of axiom (CP4) follows from

$$
\begin{aligned}
s e(P \triangleleft & (Q \triangleleft R \triangleright S) \triangleright U) \\
& =s e(Q \triangleleft R \triangleright S)[\mathrm{T} \mapsto s e(P), \mathrm{F} \mapsto s e(U)] \\
& =(s e(R)[\mathrm{T} \mapsto \operatorname{se}(Q), \mathrm{F} \mapsto s e(S)])[\mathrm{T} \mapsto s e(P), \mathrm{F} \mapsto s e(U)] \\
& =s e(R)[\mathrm{T} \mapsto s e(Q)[\mathrm{T} \mapsto s e(P), \mathrm{F} \mapsto s e(U)], \mathrm{F} \mapsto s e(S)[\mathrm{T} \mapsto s e(P), \mathrm{F} \mapsto s e(U)]] \\
& =s e(R)[\mathrm{T} \mapsto s e(P \triangleleft Q \triangleright U), \mathrm{F} \mapsto s e(P \triangleleft S \triangleright U)] \\
& =s e((P \triangleleft Q \triangleright U) \triangleleft R \triangleright(P \triangleleft S \triangleright U)) .
\end{aligned}
$$

$(\Leftarrow)$ Let $P={ }_{s e} Q$. According to Lemma 2.7 there exist basic forms $P^{\prime}$ and $Q^{\prime}$ such that $\mathrm{CP} \vdash P=P^{\prime}$ and $\mathrm{CP} \vdash Q=Q^{\prime}$, so $\mathrm{CP} \vdash P^{\prime}=Q^{\prime}$. By soundness $(\Rightarrow)$ we find $P^{\prime}={ }_{s e} Q^{\prime}$, so by Lemma 2.8, $P^{\prime}=Q^{\prime}$. Hence, $\mathrm{CP} \vdash P=P^{\prime}=Q^{\prime}=Q$.

A consequence of the above results is that for each $P \in C_{A}$ there is a unique basic form $P^{\prime}$ with $\mathrm{CP} \vdash P=P^{\prime}$, and that for each basic form, its se-image has exactly the same syntactic structure (replacing $\triangleleft$ by $\unlhd$, and $\triangleright$ by $\unrhd$ ). In the remainder of this section, we make this precise. 
Definition 2.12. The basic form function bf: $C_{A} \rightarrow B F_{A}$ is defined as follows, where $a \in A$ :

$$
\begin{aligned}
b f(\mathrm{~T}) & =\mathrm{T}, \\
b f(\mathrm{~F}) & =\mathrm{F}, \\
b f(a) & =\mathrm{T} \triangleleft a \triangleright \mathrm{F}, \\
b f(P \triangleleft Q \triangleright R) & =b f(Q)[\mathrm{T} \mapsto b f(P), \mathrm{F} \mapsto b f(R)] .
\end{aligned}
$$

Given $Q, R \in B F_{A}$, the auxiliary function $[\mathrm{T} \mapsto Q, \mathrm{~F} \mapsto R]: B F_{A} \rightarrow B F_{A}$ for which post-fix notation $P[\mathrm{~T} \mapsto Q, \mathrm{~F} \mapsto R]$ is adopted, is defined as follows:

$$
\begin{aligned}
\mathrm{T}[\mathrm{T} \mapsto Q, \mathrm{~F} \mapsto R] & =Q, \\
\mathrm{~F}[\mathrm{~T} \mapsto Q, \mathrm{~F} \mapsto R] & =R, \\
\left(P_{1} \triangleleft a \triangleright P_{2}\right)[\mathrm{T} \mapsto Q, \mathrm{~F} \mapsto R] & =P_{1}[\mathrm{~T} \mapsto Q, \mathrm{~F} \mapsto R] \triangleleft a \triangleright P_{2}[\mathrm{~T} \mapsto Q, \mathrm{~F} \mapsto R] .
\end{aligned}
$$

(The notational overloading with the leaf replacement functions on evaluation trees is harmless).

So, for given $Q, R \in B F_{A}$, the auxiliary function $[\mathrm{T} \mapsto Q, \mathrm{~F} \mapsto R]$ applied to $P \in B F_{A}$ (thus, $P[\mathrm{~T} \mapsto Q, \mathrm{~F} \mapsto R])$ replaces all $\mathrm{T}$-occurrences in $P$ by $Q$, and all F-occurrences in $P$ by $R$. The following two lemma's imply that bf is a normalization function.

Lemma 2.13. For all $P \in C_{A}, b f(P)$ is a basic form.

Proof. By structural induction. The base cases are trivial. For the inductive case we find $b f(P \triangleleft Q \triangleright R)=b f(Q)[\mathrm{T} \mapsto b f(P), \mathrm{F} \mapsto b f(R)]$, so by induction, $b f(P), b f(Q)$, and $b f(R)$ are basic forms. Furthermore, replacing all T-occurrences and F-occurrences in $b f(Q)$ by basic forms $b f(P)$ and $b f(R)$, respectively, yields a basic form.

Lemma 2.14. For each basic form $P$, bf $(P)=P$.

Proof. By structural induction on $P$.

Definition 2.15. The binary relation $=_{b f}$ on $C_{A}$ is defined as follows:

$$
P={ }_{b f} Q \Longleftrightarrow b f(P)=b f(Q) .
$$

Lemma 2.16. The relation ${ }_{b f}$ is a congruence relation.

Proof. Assume $P={ }_{b f} P^{\prime}, Q={ }_{b f} Q^{\prime}$, and $R={ }_{b f} R^{\prime}$. Then $b f(P \triangleleft Q \triangleright R)=b f(Q)[\mathrm{T} \mapsto b f(P), \mathrm{F} \mapsto$ $b f(R)]=b f\left(Q^{\prime}\right)\left[\mathrm{T} \mapsto b f\left(P^{\prime}\right), \mathrm{F} \mapsto b f\left(R^{\prime}\right)\right]=b f\left(P^{\prime} \triangleleft Q^{\prime} \triangleright R^{\prime}\right)$, so $P \triangleleft Q \triangleright R={ }_{b f} P^{\prime} \triangleleft Q^{\prime} \triangleright R^{\prime}$.

Before proving that $\mathrm{CP}$ is an axiomatization of the relation $=_{b f}$, we show that each instance of the axiom (CP4) satisfies $=_{b f}$.

Lemma 2.17. For all $P, P_{1}, P_{2}, Q_{1}, Q_{2} \in C_{A}$,

$$
b f\left(Q_{1} \triangleleft\left(P_{1} \triangleleft P \triangleright P_{2}\right) \triangleright Q_{2}\right)=b f\left(\left(Q_{1} \triangleleft P_{1} \triangleright Q_{2}\right) \triangleleft P \triangleright\left(Q_{1} \triangleleft P_{2} \triangleright Q_{2}\right)\right) .
$$

Proof. By definition, the lemma's statement is equivalent with

$$
\begin{aligned}
&(b f(P)[\mathrm{T}\left.\left.\mapsto b f\left(P_{1}\right), \mathrm{F} \mapsto b f\left(P_{2}\right)\right]\right)\left[\mathrm{T} \mapsto b f\left(Q_{1}\right), \mathrm{F} \mapsto b f\left(Q_{2}\right)\right] \\
&=b f(P)\left[\mathrm{T} \mapsto b f\left(Q_{1} \triangleleft P_{1} \triangleright Q_{2}\right), \mathrm{F} \mapsto b f\left(Q_{1} \triangleleft P_{2} \triangleright Q_{2}\right)\right] .
\end{aligned}
$$


By Lemma 2.13, $b f(P), b f\left(P_{i}\right)$, and $b f\left(Q_{i}\right)$ are basic forms. We prove (11) by structural induction on the form that $b f(P)$ can have. If $b f(P)=\mathrm{T}$, then

$$
\left(\mathrm{T}\left[\mathrm{T} \mapsto b f\left(P_{1}\right), \mathrm{F} \mapsto b f\left(P_{2}\right)\right]\right)\left[\mathrm{T} \mapsto b f\left(Q_{1}\right), \mathrm{F} \mapsto b f\left(Q_{2}\right)\right]=b f\left(P_{1}\right)\left[\mathrm{T} \mapsto b f\left(Q_{1}\right), \mathrm{F} \mapsto b f\left(Q_{2}\right)\right]
$$

and

$$
\begin{aligned}
\mathrm{T}\left[\mathrm{T} \mapsto b f\left(Q_{1} \triangleleft P_{1} \triangleright Q_{2}\right), \mathrm{F} \mapsto b f\left(Q_{1} \triangleleft P_{2} \triangleright Q_{2}\right)\right] & =b f\left(Q_{1} \triangleleft P_{1} \triangleright Q_{2}\right) \\
& =b f\left(P_{1}\right)\left[\mathrm{T} \mapsto b f\left(Q_{1}\right), \mathrm{F} \mapsto b f\left(Q_{2}\right)\right] .
\end{aligned}
$$

If $b f(P)=\mathrm{F}$, then (11) follows in a similar way.

The inductive case $b f(P)=R_{1} \triangleleft a \triangleright R_{2}$ is trivial (by definition of the last defining clause of the auxiliary functions $[\mathrm{T} \mapsto Q, \mathrm{~F} \mapsto R]$ in Definition 2.12$)$.

Theorem 2.18. For all $P, Q \in C_{A}, \mathrm{CP} \vdash P=Q \Longleftrightarrow P={ }_{b f} Q$.

Proof. $(\Rightarrow)$ By Lemma 2.16, $={ }_{b f}$ is a congruence relation and it easily follows that closed instances of the CP-axioms (CP1)-(CP3) satisfy $={ }_{b f}$. By Lemma 2.17 it follows that closed instances of axiom (CP4) also satisfy $=_{b f}$.

$(\Leftarrow)$ Assume $P={ }_{b f} Q$. According to Lemma 2.7 there exist basic forms $P^{\prime}$ and $Q^{\prime}$ such that $\mathrm{CP} \vdash P=P^{\prime}$ and $\mathrm{CP} \vdash Q=Q^{\prime}$, so $\mathrm{CP} \vdash P^{\prime}=Q^{\prime}$. By $\Rightarrow$ it follows that $P^{\prime}={ }_{b f} Q^{\prime}$, which implies by Lemma 2.14 that $P^{\prime}=Q^{\prime}$. Hence, $\mathrm{CP} \vdash P=P^{\prime}=Q^{\prime}=Q$.

Corollary 2.19. For all $P \in C_{A}, P={ }_{b f} b f(P)$ and $P={ }_{s e} b f(P)$.

Proof. By Lemma 2.13 and Lemma 2.14. $b f(P)=b f(b f(P))$, thus $P={ }_{b f} b f(P)$. By Theorem 2.18, $\mathrm{CP} \vdash P=b f(P)$, and by Theorem 2.11, $P={ }_{s e} b f(P)$.

\section{Evaluation trees for repetition-proof valuation congru- ence}

In 4 we defined repetition-proof $\mathrm{CP}$ as the extension of the axiom set $\mathrm{CP}$ with the following two axiom schemes, where $a$ ranges over $A$ :

$$
\begin{aligned}
& (x \triangleleft a \triangleright y) \triangleleft a \triangleright z=(x \triangleleft a \triangleright x) \triangleleft a \triangleright z, \\
& x \triangleleft a \triangleright(y \triangleleft a \triangleright z)=x \triangleleft a \triangleright(z \triangleleft a \triangleright z) .
\end{aligned}
$$

We write $\mathrm{CP}_{r p}(A)$ for this extension. These axiom schemes characterize that for each atom $a$, a consecutive evaluation of $a$ yields the same result, so in both cases the conditional statement at the $y$-position will not be evaluated and can be replaced by any other. Note that (CPrp1) and CPrp2 are each others dual.

We define a proper subset of basic forms with the property that each propositional statement can be proved equal to such a basic form.

Definition 3.1. Rp-basic forms are inductively defined:

- $\mathrm{T}$ and $\mathrm{F}$ are rp-basic forms, and

- $P_{1} \triangleleft a \triangleright P_{2}$ is an rp-basic form if $P_{1}$ and $P_{2}$ are rp-basic forms, and if $P_{i}$ is not equal to $\mathrm{T}$ or $\mathrm{F}$, then either the central condition in $P_{i}$ is different from $a$, or $P_{i}$ is of the form $Q_{i} \triangleleft a \triangleright Q_{i}$. 
It will turn out useful to define a function that transforms conditional statements into rp-basic forms, and that is comparable to the function $b f$.

Definition 3.2. The rp-basic form function rpbf: $C_{A} \rightarrow C_{A}$ is defined by

$$
\operatorname{rpbf}(P)=\operatorname{rpf}(b f(P)) .
$$

The auxiliary function $r p f: B F_{A} \rightarrow B F_{A}$ is defined as follows:

$$
\begin{aligned}
r p f(\mathrm{~T}) & =\mathrm{T}, \\
r p f(\mathrm{~F}) & =\mathrm{F}, \\
r p f(P \triangleleft a \triangleright Q) & =\operatorname{rpf}\left(f_{a}(P)\right) \triangleleft a \triangleright r p f\left(g_{a}(Q)\right) .
\end{aligned}
$$

For $a \in A$, the auxiliary functions $f_{a}: B F_{A} \rightarrow B F_{A}$ and $g_{a}: B F_{A} \rightarrow B F_{A}$ are defined by

$$
\begin{aligned}
f_{a}(\mathrm{~T}) & =\mathrm{T}, \\
f_{a}(\mathrm{~F}) & =\mathrm{F}, \\
f_{a}(P \triangleleft b \triangleright Q) & = \begin{cases}f_{a}(P) \triangleleft a \triangleright f_{a}(P) & \text { if } b=a, \\
P \triangleleft b \triangleright Q & \text { otherwise, }\end{cases}
\end{aligned}
$$

and

$$
\begin{aligned}
g_{a}(\mathrm{~T}) & =\mathrm{T}, \\
g_{a}(\mathrm{~F}) & =\mathrm{F}, \\
g_{a}(P \triangleleft b \triangleright Q) & = \begin{cases}g_{a}(Q) \triangleleft a \triangleright g_{a}(Q) & \text { if } b=a, \\
P \triangleleft b \triangleright Q & \text { otherwise. }\end{cases}
\end{aligned}
$$

Thus, $r p b f$ maps a conditional statement $P$ to $b f(P)$ and then transforms $b f(P)$ according to the auxiliary functions $r p f, f_{a}$, and $g_{a}$.

Lemma 3.3. For all $a \in A$ and $P \in B F_{A}$,

$$
g_{a}\left(f_{a}(P)\right)=f_{a}\left(f_{a}(P)\right)=f_{a}(P) \text { and } f_{a}\left(g_{a}(P)\right)=g_{a}\left(g_{a}(P)\right)=g_{a}(P) .
$$

Proof. By structural induction on $P$. The base cases $P \in\{\mathrm{T}, \mathrm{F}\}$ are trivial. For the inductive case $P=Q \triangleleft b \triangleright R$ we have to distinguish the cases $b=a$ and $b \neq a$. If $b=a$, then

$$
\begin{aligned}
g_{a}\left(f_{a}(Q \triangleleft a \triangleright R)\right) & =g_{a}\left(f_{a}(Q)\right) \triangleleft a \triangleright g_{a}\left(f_{a}(Q)\right) & \\
& =f_{a}(Q) \triangleleft a \triangleright f_{a}(Q) & \text { by IH } \\
& =f_{a}(Q \triangleleft a \triangleright R), &
\end{aligned}
$$

and $f_{a}\left(f_{a}(Q \triangleleft a \triangleright R)\right)=f_{a}(Q \triangleleft a \triangleright R)$ follows in a similar way. If $b \neq a$, then $f_{a}(P)=g_{a}(P)=P$, and hence $g_{a}\left(f_{a}(P)\right)=f_{a}\left(f_{a}(P)\right)=f_{a}(P)$.

The second pair of equalities can be derived in a similar way.

In order to prove that for all $P \in C_{A}, \operatorname{rpbf}(P)$ is an rp-basic form, we use the following auxiliary lemma. 
Lemma 3.4. For all $a \in A$ and $P \in B F_{A}, d(P) \geq d\left(f_{a}(P)\right)$ and $d(P) \geq d\left(g_{a}(P)\right)$.

Proof. Fix some $a \in A$. We prove these inequalities by structural induction on $P$. The base cases $P \in\{\mathrm{T}, \mathrm{F}\}$ are trivial. For the inductive case $P=Q \triangleleft b \triangleright R$ we have to distinguish the cases $b=a$ and $b \neq a$. If $b=a$, then

$$
\begin{array}{rlr}
d(Q \triangleleft a \triangleright R) & =1+\max \{d(Q), d(R)\} & \\
& \geq 1+d(Q) & \\
& \geq 1+d\left(f_{a}(Q)\right) & \text { by IH } \\
& =d\left(f_{a}(Q) \triangleleft a \triangleright f_{a}(Q)\right) & \\
& =d\left(f_{a}(Q \triangleleft a \triangleright R)\right), &
\end{array}
$$

and $d(Q \triangleleft a \triangleright R) \geq d\left(g_{a}(Q \triangleleft a \triangleright R)\right)$ follows in a similar way.

If $b \neq a$, then $f_{a}(P)=g_{a}(P)=P$, and hence $d(P) \geq d\left(f_{a}(P)\right)$ and $d(P) \geq d\left(g_{a}(P)\right)$.

Lemma 3.5. For all $P \in C_{A}, r p b f(P)$ is an rp-basic form.

Proof. We first prove an auxiliary result:

$$
\text { For all } P \in B F_{A}, r p f(P) \text { is an rp-basic form. }
$$

This follows by induction on the depth $d(P)$ of $P$. If $d(P)=0$, then $P \in\{\mathrm{T}, \mathrm{F}\}$, and hence $\operatorname{rpf}(P)=P$ is an rp-basic form. For the inductive case $d(P)=n+1$ it must be the case that $P=Q \triangleleft a \triangleright R$. We find

$$
r p f(Q \triangleleft a \triangleright R)=\operatorname{rpf}\left(f_{a}(Q)\right) \triangleleft a \triangleright r p f\left(g_{a}(R)\right),
$$

which is an rp-basic form because

- by Lemma 3.4 $f_{a}(Q)$ and $g_{a}(R)$ are basic forms with depth smaller than or equal to $n$, so by the induction hypothesis, $\operatorname{rpf}\left(f_{a}(Q)\right)$ and $\operatorname{rpf}\left(g_{a}(R)\right)$ are rp-basic forms,

- $\operatorname{rpf}\left(f_{a}(Q)\right)$ and $\operatorname{rpf}\left(g_{a}(R)\right)$ both satisfy the following property: if the central condition (if present) is $a$, then the outer arguments are equal. We show this first for $\operatorname{rpf}\left(f_{a}(Q)\right)$ by a case distinction on the form of $Q$ :

1. If $Q \in\{\mathrm{T}, \mathrm{F}\}$, then $\operatorname{rpf}\left(f_{a}(Q)\right)=Q$, so there is nothing to prove.

2. If $Q=Q_{1} \triangleleft a \triangleright Q_{2}$, then $f_{a}(Q)=f_{a}\left(Q_{1}\right) \triangleleft a \triangleright f_{a}\left(Q_{1}\right)$ and thus by Lemma 3.3 , $r p f\left(f_{a}(Q)\right)=r p f\left(f_{a}\left(Q_{1}\right)\right) \triangleleft a \triangleright r p f\left(f_{a}\left(Q_{1}\right)\right)$.

3. If $Q=Q_{1} \triangleleft b \triangleright Q_{2}$ with $b \neq a$, then $f_{a}(Q)=Q_{1} \triangleleft b \triangleright Q_{2}$, so $r p f\left(f_{a}(Q)\right)=\operatorname{rpf}\left(f_{b}\left(Q_{1}\right)\right) \triangleleft b \triangleright r p f\left(g_{b}\left(Q_{2}\right)\right)$ and there is nothing to prove.

The fact that $\operatorname{rpf}\left(g_{a}(R)\right)$ satisfies this property follows in a similar way.

This finishes the proof of (2).

The lemma's statement now follows by structural induction: the base cases (comprising a single atom $a$ ) are again trivial, and for the inductive case,

$$
\operatorname{rpbf}(P \triangleleft Q \triangleright R)=\operatorname{rpf}(b f(P \triangleleft Q \triangleright R))=\operatorname{rpf}(S)
$$

for some basic form $S$ by Lemma 2.13, and by auxiliary result (2), $r p f(S)$ is an rp-basic form. 
The following, somewhat technical result is used in Proposition 3.7 and Lemma 3.8 .

Lemma 3.6. If $Q \triangleleft a \triangleright R$ is an rp-basic form, then $Q=\operatorname{rpf}(Q)=\operatorname{rpf}\left(f_{a}(Q)\right)$ and $R=\operatorname{rpf}(R)=$ $\operatorname{rpf}\left(g_{a}(R)\right)$.

Proof. We first prove an auxiliary result:

$$
\text { If } Q \triangleleft a \triangleright R \text { is an rp-basic form, then } f_{a}(Q)=g_{a}(Q) \text { and } f_{a}(R)=g_{a}(R) \text {. }
$$

We prove both equalities by simultaneous induction on the structure of $Q$ and $R$. The base case, thus $Q, R \in\{\mathrm{T}, \mathrm{F}\}$, is trivial. If $Q=Q_{1} \triangleleft a \triangleright Q_{1}$ and $R=R_{1} \triangleleft a \triangleright R_{1}$, then $Q$ and $R$ are rp-basic forms with central condition $a$, so

$$
\begin{aligned}
f_{a}(Q) & =f_{a}\left(Q_{1}\right) \triangleleft a \triangleright f_{a}\left(Q_{1}\right) & & \\
& =g_{a}\left(Q_{1}\right) \triangleleft a \triangleright g_{a}\left(Q_{1}\right) & & \text { by IH } \\
& =g_{a}(Q), & &
\end{aligned}
$$

and the equality for $R$ follows in a similar way. If $Q=Q_{1} \triangleleft a \triangleright Q_{1}$ and $R \neq R_{1} \triangleleft a \triangleright R_{1}$, then $f_{a}(R)=g_{a}(R)=R$, and the result follows as above. All remaining cases follow in a similar way, which finishes the proof of (3).

We now prove the lemma's statement by simultaneous induction on the structure of $Q$ and $R$. The base case, thus $Q, R \in\{\mathrm{T}, \mathrm{F}\}$, is again trivial. If $Q=Q_{1} \triangleleft a \triangleright Q_{1}$ and $R=R_{1} \triangleleft a \triangleright R_{1}$, then by auxiliary result (즈),

$$
\operatorname{rpf}(Q)=\operatorname{rpf}\left(f_{a}\left(Q_{1}\right)\right) \triangleleft a \triangleright \operatorname{rpf}\left(f_{a}\left(Q_{1}\right)\right),
$$

and by induction, $Q_{1}=\operatorname{rpf}\left(Q_{1}\right)=\operatorname{rpf}\left(f_{a}\left(Q_{1}\right)\right)$. Hence, $\operatorname{rpf}(Q)=Q_{1} \triangleleft a \triangleright Q_{1}$, and

$$
\begin{array}{rlr}
\operatorname{rpf}\left(f_{a}(Q)\right) & =\operatorname{rpf}\left(f_{a}\left(f_{a}\left(Q_{1}\right)\right)\right) \triangleleft a \triangleright r p f\left(g_{a}\left(f_{a}\left(Q_{1}\right)\right)\right) & \\
& =\operatorname{rpf}\left(f_{a}\left(Q_{1}\right)\right) \triangleleft a \triangleright \operatorname{rpf}\left(f_{a}\left(Q_{1}\right)\right) & \text { by Lemma } 3.3 \\
& =Q_{1} \triangleleft a \triangleright Q_{1}, &
\end{array}
$$

and the equalities for $R$ follow in a similar way.

If $Q=Q_{1} \triangleleft a \triangleright Q_{1}$ and $R \neq R_{1} \triangleleft a \triangleright R_{1}$, the lemma's equalities follow in a similar way, although a bit simpler because $g_{a}(R)=f_{a}(R)=R$.

For all remaining cases, the lemma's equalities follow in a similar way.

Proposition 3.7 ( $r p b f$ is a normalization function). For all $P \in C_{A}$, rpbf( $P$ ) is an rp-basic form, and for each rp-basic form $P, \operatorname{rpbf}(P)=P$.

Proof. The first statement is Lemma 3.5. For the second statement, it suffices by Lemma 2.14] to prove that for each rp-basic form $P, \operatorname{rpf}(P)=P$. This follows by case distinction on $P$. The cases $P \in\{\mathrm{T}, \mathrm{F}\}$ follow immediately, and otherwise $P=Q \triangleleft a \triangleright R$, and thus $\operatorname{rpf}(P)=$ $r p f\left(f_{a}(Q)\right) \triangleleft a \triangleright r p f\left(g_{a}(R)\right)$. By Lemma 3.6. $\operatorname{rpf}\left(f_{a}(Q)\right)=Q$ and $\operatorname{rpf}\left(g_{a}(R)\right)=R$, hence $\operatorname{rpf}(P)=P$.

Lemma 3.8. For all $P \in B F_{A}, \mathrm{CP}_{r p}(A) \vdash P=\operatorname{rpf}(P)$.

Proof. We apply structural induction on $P$. The base cases $P \in\{\mathrm{T}, \mathrm{F}\}$ are trivial. Assume $P=P_{1} \triangleleft a \triangleright P_{2}$. By induction $\mathrm{CP}_{r p}(A) \vdash P_{i}=\operatorname{rpf}\left(P_{i}\right)$. We proceed by a case distinction on the form that $P_{1}$ and $P_{2}$ can have: 
1. If $P_{i} \in\left\{\mathrm{T}, \mathrm{F}, Q_{i} \triangleleft b_{i} \triangleright Q_{i}^{\prime}\right\}$ with $b_{i} \neq a$, then $f_{a}\left(P_{1}\right)=P_{1}$ and $g_{a}\left(P_{2}\right)=P_{2}$, and hence $r p f(P)=\operatorname{rpf}\left(P_{1}\right) \triangleleft a \triangleright r p f\left(P_{2}\right)$, and thus $\mathrm{CP}_{r p}(A) \vdash P=\operatorname{rpf}(P)$.

2. If $P_{1}=R_{1} \triangleleft a \triangleright R_{2}$ and $P_{2} \in\left\{\mathrm{T}, \mathrm{F}, Q^{\prime} \triangleleft b \triangleright Q^{\prime \prime}\right\}$ with $b \neq a$, then $g_{a}\left(P_{2}\right)=P_{2}$ and by auxiliary result (2) in the proof of Lemma 3.5. $\operatorname{rpf}\left(R_{1}\right)$ and $\operatorname{rpf}\left(P_{2}\right)$ are rp-basic forms. We derive

$$
\begin{aligned}
\mathrm{CP}_{r p}(A) & \vdash P=\left(R_{1} \triangleleft a \triangleright R_{2}\right) \triangleleft a \triangleright P_{2} & & \\
& =\left(R_{1} \triangleleft a \triangleright R_{1}\right) \triangleleft a \triangleright P_{2} & & \text { by CPrp1 } \\
& =\left(r p f\left(R_{1}\right) \triangleleft a \triangleright r p f\left(R_{1}\right)\right) \triangleleft a \triangleright r p f\left(P_{2}\right) & & \text { by IH } \\
& =\left(r p f\left(f_{a}\left(R_{1}\right)\right) \triangleleft a \triangleright r p f\left(f_{a}\left(R_{1}\right)\right) \triangleleft a \triangleright r p f\left(g_{a}\left(P_{2}\right)\right)\right. & & \text { by Lemma 3.6 } \\
& =r p f\left(f_{a}\left(R_{1} \triangleleft a \triangleright R_{2}\right)\right) \triangleleft a \triangleright r p f\left(g_{a}\left(P_{2}\right)\right) & & \\
& =r p f\left(\left(R_{1} \triangleleft a \triangleright R_{2}\right) \triangleleft a \triangleright P_{2}\right) & & \\
& =r p f(P) . & &
\end{aligned}
$$

3. If $P_{1} \in\left\{\mathrm{T}, \mathrm{F}, Q^{\prime} \triangleleft b \triangleright Q^{\prime \prime}\right\}$ with $b \neq a$ and $P_{2}=S_{1} \triangleleft a \triangleright S_{2}$, we can proceed as in the previous case, but now using axiom scheme CPrp2 and the identity $f_{a}\left(P_{1}\right)=P_{1}$, and the fact that $\operatorname{rpf}\left(P_{1}\right)$ and $\operatorname{rpf}\left(S_{2}\right)$ are rp-basic forms.

4. If $P_{1}=R_{1} \triangleleft a \triangleright R_{2}$ and $P_{2}=S_{1} \triangleleft a \triangleright S_{2}$, we can proceed as in two previous cases, now using both (CPrp1) and (CPrp2), and the fact that $\operatorname{rpf}\left(R_{1}\right)$ and $\operatorname{rpf}\left(S_{2}\right)$ are rp-basic forms.

Theorem 3.9. For all $P \in C_{A}, \mathrm{CP}_{r p}(A) \vdash P=r p b f(P)$.

Proof. By Theorem 2.18 and Corollary 2.19 we find $\mathrm{CP}_{r p}(A) \vdash P=b f(P)$. By Lemma 3.8, $\mathrm{CP}_{r p}(A) \vdash b f(P)=\operatorname{rpf}(b f(P))$, and $\operatorname{rpf}(b f(P))=\operatorname{rpbf}(P)$.

Definition 3.10. The binary relation $=_{r p b f}$ on $C_{A}$ is defined as follows:

$$
P={ }_{r p b f} Q \Longleftrightarrow \operatorname{rpbf}(P)=\operatorname{rpbf}(Q) .
$$

Theorem 3.11. For all $P, Q \in C_{A}, \mathrm{CP}_{r p}(A) \vdash P=Q \Longleftrightarrow P={ }_{r p b f} Q$.

Proof. $(\Rightarrow)$ Assume $\mathrm{CP}_{r p}(A) \vdash P=Q$. By Theorem 3.9, $\mathrm{CP}_{r p}(A) \vdash \operatorname{rpbf}(P)=\operatorname{rpbf}(Q)$. In [4] the following two statements are proved (Theorem 6.3 and an auxiliary result in its proof), where ${ }_{r p}$ is a binary relation on $C_{A}$ :

1. For all $P, Q \in C_{A}, \quad \mathrm{CP}_{r p}(A) \vdash P=Q \Longleftrightarrow P={ }_{r p} Q$.

2. For all rp-basic forms $P$ and $Q, \quad P={ }_{r p} Q \Rightarrow P=Q$.

By Lemma 3.5 these statements imply $r p b f(P)=r p b f(Q)$, that is, $P={ }_{r p b f} Q$.

$(\Leftarrow)$ Assume $P={ }_{r p b f} Q$. By Theorem $\left[3.9\right.$, $\mathrm{CP}_{r p}(A) \vdash P=Q$.

So, the relation $=_{r p b f}$ is axiomatized by $\mathrm{CP}_{r p}(A)$, and is thus a congruence. With this observation in mind, we define a transformation on evaluation trees that mimics the function $r p b f$, and prove that equality of two such transformed trees characterizes the congruence that is axiomatized by $\mathrm{CP}_{r p}(A)$. 
Definition 3.12. The unary repetition-proof evaluation function

$$
\text { rpse }: C_{A} \rightarrow \mathcal{T}_{A}
$$

yields repetition-proof evaluation trees and is defined by

$$
\operatorname{rpse}(P)=\operatorname{rp}(\operatorname{se}(P)) \text {. }
$$

The auxiliary function $r p: \mathcal{T}_{A} \rightarrow \mathcal{T}_{A}$ is defined as follows $(a \in A)$ :

$$
\begin{aligned}
r p(\mathrm{~T}) & =\mathrm{T}, \\
r p(\mathrm{~F}) & =\mathrm{F}, \\
r p(X \unlhd a \unrhd Y) & =r p\left(F_{a}(X)\right) \unlhd a \unrhd r p\left(G_{a}(Y)\right) .
\end{aligned}
$$

For $a \in A$, the auxiliary functions $F_{a}: \mathcal{T}_{A} \rightarrow \mathcal{T}_{A}$ and $G_{a}: \mathcal{T}_{A} \rightarrow \mathcal{T}_{A}$ are defined by

$$
\begin{aligned}
F_{a}(\mathrm{~T}) & =\mathrm{T}, \\
F_{a}(\mathrm{~F}) & =\mathrm{F}, \\
F_{a}(X \unlhd b \unrhd Y) & = \begin{cases}F_{a}(X) \unlhd a \unrhd F_{a}(X) & \text { if } b=a, \\
X \unlhd b \unrhd Y & \text { otherwise, }\end{cases}
\end{aligned}
$$

and

$$
\begin{aligned}
G_{a}(\mathrm{~T}) & =\mathrm{T}, \\
G_{a}(\mathrm{~F}) & =\mathrm{F}, \\
G_{a}(X \unlhd b \unrhd Y) & = \begin{cases}G_{a}(Y) \unlhd a \unrhd G_{a}(Y) & \text { if } b=a, \\
X \unlhd b \unrhd Y & \text { otherwise. }\end{cases}
\end{aligned}
$$

Example 3.13. Let $P=a \triangleleft(\mathrm{F} \triangleleft a \triangleright \mathrm{T}) \triangleright \mathrm{F}$. We depict $s e(P)$ (as in Example 2.4) and the repetition-proof evaluation tree $\operatorname{rpse}(P)=\mathrm{F} \unlhd a \unrhd(\mathrm{F} \unlhd a \unrhd \mathrm{F})$ :
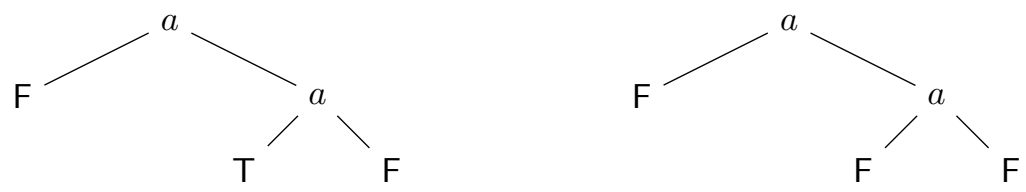

End example.

The similarities between rpse and the function rpbf can be exploited: We use the following lemma in the proof of this section's last completeness result.

Lemma 3.14. For all $P \in B F_{A}, r p(s e(P))=\operatorname{se}(r p f(P))$.

Proof. We first prove an auxiliary result:

For all $a \in A$ and $P \in B F_{A}, F_{a}(s e(P))=\operatorname{se}\left(f_{a}(P)\right)$ and $G_{a}(\operatorname{se}(P))=\operatorname{se}\left(g_{a}(P)\right)$.

Fix some $a \in A$. We prove (4) by structural induction on $P$. The base cases $P \in\{\mathrm{T}, \mathrm{F}\}$ are trivial. For the inductive case $P=Q \triangleleft b \triangleright R$ we have to distinguish the cases $b=a$ and $b \neq a$. If 
$b=a$, then

$$
\begin{aligned}
F_{a}(\operatorname{se}(Q \triangleleft a \triangleright R)) & =F_{a}(\operatorname{se}(Q) \unlhd a \unrhd s e(R)) \\
& =F_{a}(\operatorname{se}(Q)) \unlhd a \unrhd F_{a}(\operatorname{se}(Q)) \\
& =\operatorname{se}\left(f_{a}(Q)\right) \unlhd a \unrhd \operatorname{se}\left(f_{a}(Q)\right) \\
& =\operatorname{se}\left(f_{a}(Q \triangleleft a \triangleright R)\right),
\end{aligned}
$$

and if $b \neq a$, then

$$
\begin{aligned}
F_{a}(\operatorname{se}(Q \triangleleft b \triangleright R)) & =F_{a}(\operatorname{se}(Q) \unlhd b \unrhd s e(R)) \\
& =\operatorname{se}(Q) \unlhd b \unrhd s e(R) \\
& =\operatorname{se}(Q \triangleleft b \triangleright R) \\
& =\operatorname{se}\left(f_{a}(Q \triangleleft b \triangleright R)\right) .
\end{aligned}
$$

The second equality can be derived in a similar way, and this finishes the proof of (44).

We prove the lemma's statement by induction on $d(P)$. The base cases $P \in\{\mathrm{T}, \mathrm{F}\}$ follow immediately. Assume $P=Q \triangleleft a \triangleright R$, then

$$
\begin{array}{rlrl}
r p(\operatorname{se}(Q \triangleleft a \triangleright R)) & =r p(\operatorname{se}(Q) \unlhd a \unrhd s e(R)) & & \\
& =r p\left(F_{a}(\operatorname{se}(Q))\right) \unlhd a \unrhd r p\left(G_{a}(\operatorname{se}(R))\right) & & \\
& =r p\left(\operatorname{se}\left(f_{a}(Q)\right)\right) \unlhd a \unrhd r p\left(\operatorname{se}\left(g_{a}(R)\right)\right) & & \text { by (4) } \\
& =\operatorname{se}\left(\operatorname{rpf}\left(f_{a}(Q)\right)\right) \unlhd a \unrhd \operatorname{se}\left(\operatorname{rpf}\left(g_{a}(R)\right)\right) & & \text { by IH (and Lemma 3.4) } \\
& =\operatorname{se}\left(\operatorname{rpf}\left(f_{a}(Q)\right) \triangleleft a \triangleright r p f\left(g_{a}(R)\right)\right) & & \\
& =\operatorname{se}(\operatorname{rpf}(Q \triangleleft a \triangleright R)) .
\end{array}
$$

Finally, we relate conditional statements by means of their repetition-proof evaluation trees.

Definition 3.15. Repetition-proof valuation congruence, notation $=_{r p s e}$, is defined on $C_{A}$ as follows:

$$
P={ }_{\text {rpse }} Q \Longleftrightarrow \operatorname{rpse}(P)=\operatorname{rpse}(Q) .
$$

The following characterization result immediately implies that $=_{r p s e}$ is a congruence relation on $C_{A}$ (and hence justifies calling it a congruence).

Proposition 3.16. For all $P, Q \in C_{A}, P={ }_{r p s e} Q \Longleftrightarrow P={ }_{r p b f} Q$.

Proof. $(\Rightarrow)$ Assume rpse $(P)=\operatorname{rpse}(Q)$, thus $r p(s e(P))=r p(\operatorname{se}(Q))$. By Corollary 2.19 , $r p(s e(b f(P)))=r p(s e(b f(Q)))$, so by Lemma 3.14, $s e(r p f(b f(P)))=\operatorname{se}(r p f(b f(Q)))$.

By Lemma 2.8 and auxiliary result (2) (see the proof of Lemma 3.5), it follows that $\operatorname{rpf}(b f(P))=$ $r p f(b f(Q))$, that is, $P={ }_{r p b f} Q$.

$(\Leftarrow)$ Assume $P={ }_{r p b f} Q$, thus $\operatorname{rpf}(b f(P))=\operatorname{rpf}(b f(Q))$ and $\operatorname{se}(\operatorname{rpf}(b f(P)))=\operatorname{se}(\operatorname{rpf}(b f(Q)))$. By Lemma 3.14, $r p(s e(b f(P)))=r p(\operatorname{se}(b f(Q)))$. By Corollary 2.19, $s e(b f(P))=s e(P)$ and $s e(b f(Q))=s e(Q)$, so $r p(s e(P))=r p(s e(Q))$, that is, $P={ }_{r p s e} Q$.

We end this section with the completeness result we were after.

Theorem 3.17 (Completeness of $\mathrm{CP}_{r p}(A)$ ). For all $P, Q \in C_{A}$,

$$
\mathrm{CP}_{r p}(A) \vdash P=Q \Longleftrightarrow P={ }_{r p s e} Q
$$

Proof. Combine Theorem 3.11 and Proposition 3.16 . 


\section{Evaluation trees for contractive valuation congruence}

In [4] we introduced $\mathrm{CP}_{c r}(A)$, contractive $\mathrm{CP}$, as the extension of $\mathrm{CP}$ with the following two axiom schemes, where $a$ ranges over $A$ :

$$
\begin{aligned}
& (x \triangleleft a \triangleright y) \triangleleft a \triangleright z=x \triangleleft a \triangleright z, \\
& x \triangleleft a \triangleright(y \triangleleft a \triangleright z)=x \triangleleft a \triangleright z .
\end{aligned}
$$

These schemes prescribe contraction for each atom $a$ for respectively the true-case and the falsecase, and are each others dual. It easily follows that the axiom schemes CPrp1 and (CPrp2 are derivable from $\mathrm{CP}_{c r}(A)$, so $\mathrm{CP}_{c r}(A)$ is also an axiomatic extension of $\mathrm{CP}_{r p}(A)$.

Again, we define a proper subset of basic forms with the property that each propositional statement can be proved equal to such a basic form.

Definition 4.1. Cr-basic forms are inductively defined:

- $\mathrm{T}$ and $\mathrm{F}$ are cr-basic forms, and

- $P_{1} \triangleleft a \triangleright P_{2}$ is a cr-basic form if $P_{1}$ and $P_{2}$ are cr-basic forms, and if $P_{i}$ is not equal to $\mathrm{T}$ or $\mathrm{F}$, the central condition in $P_{i}$ is different from a.

It will turn out useful to define a function that transforms conditional statements into cr-basic forms, and that is comparable to the function bf (see Definition 2.12).

Definition 4.2. The cr-basic form function cbf: $C_{A} \rightarrow C_{A}$ is defined by

$$
c b f(P)=c f(b f(P)) .
$$

The auxiliary function $\mathrm{cf}: B F_{A} \rightarrow B F_{A}$ is defined as follows:

$$
\begin{aligned}
c f(\mathrm{~T}) & =\mathrm{T} \\
c f(\mathrm{~F}) & =\mathrm{F}, \\
c f(P \triangleleft a \triangleright Q) & =c f\left(h_{a}(P)\right) \triangleleft a \triangleright c f\left(j_{a}(Q)\right) .
\end{aligned}
$$

For $a \in A$, the auxiliary functions $h_{a}: B F_{A} \rightarrow B F_{A}$ and $j_{a}: B F_{A} \rightarrow B F_{A}$ are defined by

$$
\begin{aligned}
h_{a}(\mathrm{~T}) & =\mathrm{T}, \\
h_{a}(\mathrm{~F}) & =\mathrm{F}, \\
h_{a}(P \triangleleft b \triangleright Q) & = \begin{cases}h_{a}(P) & \text { if } b=a, \\
P \triangleleft b \triangleright Q & \text { otherwise, }\end{cases}
\end{aligned}
$$

and

$$
\begin{aligned}
j_{a}(\mathrm{~T}) & =\mathrm{T}, \\
j_{a}(\mathrm{~F}) & =\mathrm{F}, \\
j_{a}(P \triangleleft b \triangleright Q) & = \begin{cases}j_{a}(Q) & \text { if } b=a, \\
P \triangleleft b \triangleright Q & \text { otherwise. }\end{cases}
\end{aligned}
$$

Thus, $c b f$ maps a conditional statement $P$ to $b f(P)$ and then transforms $b f(P)$ according to the auxiliary functions $c f, h_{a}$, and $j_{a}$. 
Lemma 4.3. For all $a \in A$ and $P \in B F_{A}, d(P) \geq d\left(h_{a}(P)\right)$ and $d(P) \geq d\left(j_{a}(P)\right)$.

Proof. Fix some $a \in A$. We prove these inequalities by structural induction on $P$. The base cases $P \in\{\mathrm{T}, \mathrm{F}\}$ are trivial. For the inductive case $P=Q \triangleleft b \triangleright R$ we have to distinguish the cases $b=a$ and $b \neq a$. If $b=a$, then

$$
\begin{array}{rlrl}
d(Q \triangleleft a \triangleright R) & =1+\max \{d(Q), d(R)\} & & \\
& \geq 1+d(Q) & \\
& \geq 1+d\left(h_{a}(Q)\right) & \text { by IH } \\
& =1+d\left(h_{a}(Q \triangleleft a \triangleright R)\right), &
\end{array}
$$

and $d(Q \triangleleft a \triangleright R) \geq d\left(j_{a}(Q \triangleleft a \triangleright R)\right)$ follows in a similar way.

If $b \neq a$, then $h_{a}(P)=j_{a}(P)=P$, and hence $d(P) \geq d\left(h_{a}(P)\right)$ and $d(P) \geq d\left(j_{a}(P)\right)$.

Lemma 4.4. For all $P \in C_{A}, \operatorname{cbf}(P)$ is a cr-basic form.

Proof. We first prove an auxiliary result:

$$
\text { For all } P \in B F_{A}, c f(P) \text { is a cr-basic form. }
$$

This follows by induction on the depth $d(P)$ of $P$. If $d(P)=0$, then $P \in\{\mathrm{T}, \mathrm{F}\}$, and hence $c f(P)=P$ is a cr-basic form. For the inductive case $d(P)=n+1$ it must be the case that $P=Q \triangleleft a \triangleright R$. We find

$$
c f(Q \triangleleft a \triangleright R)=c f\left(h_{a}(Q)\right) \triangleleft a \triangleright c f\left(j_{a}(R)\right),
$$

which is a cr-basic form because

- by Lemma 4.3 $h_{a}(Q)$ and $j_{a}(R)$ are basic forms with depth smaller than or equal to $n$, so by the induction hypothesis, $c f\left(h_{a}(Q)\right)$ and $c f\left(j_{a}(R)\right)$ are cr-basic forms,

- by definition of the auxiliary functions $h_{a}$ and $j_{a}$, the central condition of $h_{a}(Q)$ and $j_{a}(R)$ is not equal to $a$, hence $c f\left(h_{a}(Q)\right) \triangleleft a \triangleright c f\left(j_{a}(R)\right)$ is a cr-basic form.

This completes the proof of (5).

The lemma's statement now follows by structural induction: the base cases (comprising a single atom $a$ ) are again trivial, and for the inductive case,

$$
c b f(P \triangleleft Q \triangleright R)=c f(b f(P \triangleleft Q \triangleright R))=c f(S)
$$

for some basic form $S$ by Lemma 2.13, and by (5), $c f(S)$ is a cr-basic form.

The following, somewhat technical lemma is used in Proposition 4.6 and Lemma 4.7.

Lemma 4.5. If $Q \triangleleft a \triangleright R$ is a cr-basic form, then $Q=c f(Q)=c f\left(h_{a}(Q)\right)$ and $R=c f(R)=$ $c f\left(j_{a}(R)\right)$.

Proof. By simultaneous induction on the structure of $Q$ and $R$. The base case, thus $Q, R \in\{\mathrm{T}, \mathrm{F}\}$, is again trivial. If $Q=Q_{1} \triangleleft b \triangleright Q_{2}$ and $R=R_{1} \triangleleft c \triangleright R_{2}$, then $b \neq a \neq c$ and thus $h_{a}(Q)=Q$ and $j_{a}(R)=R$. Moreover, $Q_{1}$ and $Q_{2}$ have no central condition $b$, hence $h_{b}\left(Q_{1}\right)=Q_{1}$ and $j_{b}\left(Q_{2}\right)=Q_{2}$, and thus

$$
\begin{aligned}
c f(Q) & =c f\left(h_{b}\left(Q_{1}\right)\right) \triangleleft b \triangleright c f\left(j_{b}\left(Q_{2}\right)\right) \\
& =c f\left(Q_{1}\right) \triangleleft b \triangleright c f\left(Q_{2}\right) \\
& =Q_{1} \triangleleft b \triangleright Q_{2} .
\end{aligned}
$$

by IH 
The equalities for $R$ follow in a similar way.

If $Q=Q_{1} \triangleleft b \triangleright Q_{1}$ and $R \in\{\mathrm{T}, \mathrm{F}\}$, the lemma's equalities follow in a similar way, and this is also the case if $Q \in\{\mathrm{T}, \mathrm{F}\}$ and $R=Q_{1} \triangleleft b \triangleright Q_{1}$.

With Lemma 4.5 we can easily prove the following result.

Proposition 4.6 ( $c b f$ is a normalization function). For each $P \in C_{A}, c b f(P)$ is a cr-basic form, and for each cr-basic form $P, \operatorname{cbf}(P)=P$.

Proof. The first statement is Lemma 4.4. For the second statement, it suffices by Lemma 2.14 to prove that $c f(P)=P$. We prove this by case distinction on $P$. The cases $P \in\{\mathrm{T}, \mathrm{F}\}$ follow immediately, and otherwise $P=Q \triangleleft a \triangleright R$, and thus $c f(P)=c f\left(h_{a}(Q)\right) \triangleleft a \triangleright c f\left(j_{a}(R)\right)$. By Lemma 4.5, $c f\left(h_{a}(Q)\right)=Q$ and $c f\left(j_{a}(R)\right)=R$, hence $c f(P)=P$.

Lemma 4.7. For all $P \in B F_{A}, \mathrm{CP}_{c r}(A) \vdash P=c f(P)$.

Proof. We first prove two auxiliary results:

$$
\begin{aligned}
\text { For all } a \in A \text { and } P, Q \in B F_{A}, & \mathrm{CP}_{c r}(A) \vdash P \triangleleft a \triangleright Q \\
\mathrm{CP}_{c r}(A) \vdash P \triangleleft a \triangleright Q & =P \triangleright j_{a}(Q),
\end{aligned}
$$

Fix some $a \in A$. We prove (6) by structural induction on $Q$. The base cases $Q \in\{\mathrm{T}, \mathrm{F}\}$ are trivial. For the inductive case $Q=Q_{1} \triangleleft b \triangleright Q_{2}$ we have to distinguish the cases $b=a$ and $b \neq a$. If $b=a$, then $j_{a}(Q)=j_{a}\left(Q_{2}\right)$ and

$$
\begin{aligned}
\mathrm{CP}_{c r}(A) \vdash P \triangleleft a \triangleright\left(Q_{1} \triangleleft a \triangleright Q_{2}\right) & =P \triangleleft a \triangleright Q_{2} & & \text { by (CPcr2) } \\
& =P \triangleleft a \triangleright j_{a}\left(Q_{2}\right) & & \text { by IH } \\
& =P \triangleleft a \triangleright j_{a}(Q) . & &
\end{aligned}
$$

If $b \neq a$ then $j_{a}(Q)=Q$, hence $\mathrm{CP}_{c r}(A) \vdash P \triangleleft a \triangleright Q=P \triangleleft a \triangleright j_{a}(Q)$.

Auxiliary result (7) follows in a similar way by structural induction on $P$ and with help of axiom scheme (CPcr1).

The lemma's statement follows by induction on $d(P)$. The base cases $P \in\{\mathrm{T}, \mathrm{F}\}$ are trivial. For the inductive case, assume $P=Q \triangleleft a \triangleright R$. We derive

$$
\begin{aligned}
& \mathrm{CP}_{c r}(A) \vdash Q \triangleleft a \triangleright R=h_{a}(Q) \triangleleft a \triangleright j_{a}(R) \quad \text { by (6), (7) } \\
& =c f\left(h_{a}(Q)\right) \triangleleft a \triangleright c f\left(j_{a}(R)\right) \quad \text { by IH (and Lemma 4.3) } \\
& =c f(Q \triangleleft a \triangleright R) \text {. }
\end{aligned}
$$

Theorem 4.8. For all $P \in C_{A}, \mathrm{CP}_{c r}(A) \vdash P=\operatorname{cbf}(P)$.

Proof. By Theorem 2.18 and Corollary 2.19, $\mathrm{CP}_{c r}(A) \vdash P=b f(P)$, and by Lemma 4.7 , $\mathrm{CP}_{c r}(A) \vdash b f(P)=c f(b f(P))$, and $c f(b f(P))=c b f(P)$.

Definition 4.9. The binary relation $={ }_{c b f}$ on $C_{A}$ is defined as follows:

$$
P={ }_{c b f} Q \Longleftrightarrow \operatorname{cbf}(P)=\operatorname{cbf}(Q) .
$$


Theorem 4.10. For all $P, Q \in C_{A}, \mathrm{CP}_{c r}(A) \vdash P=Q \Longleftrightarrow P={ }_{c b f} Q$.

Proof. $(\Rightarrow)$ Assume $\mathrm{CP}_{c r}(A) \vdash P=Q$. Then, by Theorem 4.8 $\mathrm{CP}_{c r}(A) \vdash \operatorname{cbf}(P)=\operatorname{cbf}(Q)$. In [4] the following two statements are proved (Theorem 6.4 and an auxiliary result in its proof), where $=_{c r}$ is a binary relation on $C_{A}$ :

1. For all $P, Q \in C_{A}, \quad \mathrm{CP}_{c r}(A) \vdash P=Q \Longleftrightarrow P={ }_{c r} Q$.

2. For all cr-basic forms $P$ and $Q, \quad P={ }_{c r} Q \Rightarrow P=Q$.

By Lemma 4.4, these statements imply $\operatorname{cbf}(P)=\operatorname{cbf}(Q)$, that is, $P={ }_{c b f} Q$.

$(\Leftarrow)$ Assume $P={ }_{c b f} Q$. By Theorem 4.8, $\mathrm{CP}_{c r}(A) \vdash P=Q$.

Hence, the relation $=_{c b f}$ is axiomatized by $\mathrm{CP}_{c r}(A)$, and is thus a congruence. We now define a transformation on evaluation trees that mimics the function $c b f$, and prove that equality of two such transformed trees characterizes the congruence that is axiomatized by $\mathrm{CP}_{c r}(A)$.

Definition 4.11. The unary contractive evaluation function

$$
\text { cse }: C_{A} \rightarrow \mathcal{T}_{A}
$$

yields contractive evaluation trees and is defined by

$$
\operatorname{cse}(P)=\operatorname{cr}(\operatorname{se}(P)) .
$$

The auxiliary function $\mathrm{cr}: \mathcal{T}_{A} \rightarrow \mathcal{T}_{A}$ is defined as follows $(a \in A)$ :

$$
\begin{aligned}
\operatorname{cr}(\mathrm{T}) & =\mathrm{T}, \\
\operatorname{cr}(\mathrm{F}) & =\mathrm{F}, \\
\operatorname{cr}(X \unlhd a \unrhd Y) & =\operatorname{cr}\left(H_{a}(X)\right) \unlhd a \unrhd \operatorname{cr}\left(J_{a}(Y)\right) .
\end{aligned}
$$

For $a \in A$, the auxiliary functions $H_{a}: \mathcal{T}_{A} \rightarrow \mathcal{T}_{A}$ and $J_{a}: \mathcal{T}_{A} \rightarrow \mathcal{T}_{A}$ are defined by

$$
\begin{aligned}
H_{a}(\mathrm{~T}) & =\mathrm{T}, \\
H_{a}(\mathrm{~F}) & =\mathrm{F}, \\
H_{a}(X \unlhd b \unrhd Y) & = \begin{cases}H_{a}(X) & \text { if } b=a, \\
X \unlhd b \unrhd Y & \text { otherwise, }\end{cases}
\end{aligned}
$$

and

$$
\begin{aligned}
J_{a}(\mathrm{~T}) & =\mathrm{T}, \\
J_{a}(\mathrm{~F}) & =\mathrm{F}, \\
J_{a}(X \unlhd b \unrhd Y) & = \begin{cases}J_{a}(Y) & \text { if } b=a, \\
X \unlhd b \unrhd Y & \text { otherwise. }\end{cases}
\end{aligned}
$$

As a simple example we depict $s e((a \triangleleft a \triangleright \mathrm{F}) \triangleleft a \triangleright \mathrm{F})$ and the contractive evaluation tree $\operatorname{cse}((a \triangleleft a \triangleright \mathrm{F}) \triangleleft a \triangleright \mathrm{F})$ :
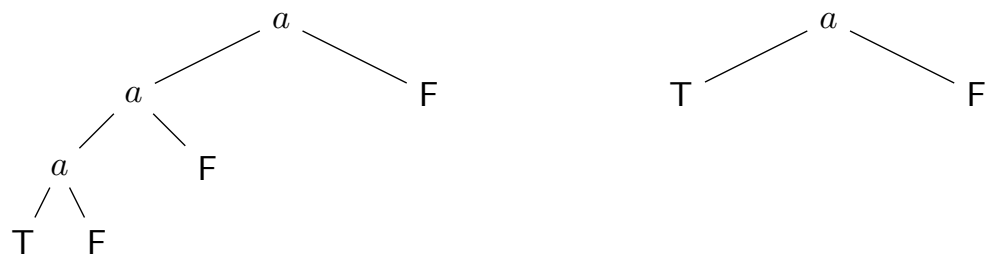
The similarities between the evaluation function cse and the function $c b f$ can be exploited, and we use the following lemma in the proof of the next completeness result.

Lemma 4.12. For all $P \in B F_{A}, \operatorname{cr}(\operatorname{se}(P))=\operatorname{se}(\operatorname{cf}(P))$.

Proof. We first prove the following auxiliary result:

For all $a \in A$ and $P \in B F_{A}, H_{a}(s e(P))=s e\left(h_{a}(P)\right)$ and $J_{a}(s e(P))=s e\left(j_{a}(P)\right)$.

Fix some $a \in A$. We prove (8) by structural induction on $P$. The base cases $P \in\{\mathrm{T}, \mathrm{F}\}$ are trivial. For the inductive case $P=Q \triangleleft b \triangleright R$ we have to distinguish the cases $b=a$ and $b \neq a$. If $b=a$, then

$$
\begin{aligned}
H_{a}(\operatorname{se}(Q \triangleleft a \triangleright R)) & =H_{a}(\operatorname{se}(Q) \unlhd a \unrhd s e(R)) \\
& =H_{a}(\operatorname{se}(Q)) \\
& =\operatorname{se}\left(h_{a}(Q)\right) \\
& =\operatorname{se}\left(h_{a}(Q \triangleleft a \triangleright R)\right),
\end{aligned}
$$

by $\mathrm{IH}$

and if $b \neq a$, then

$$
\begin{aligned}
H_{a}(\operatorname{se}(Q \triangleleft b \triangleright R)) & =H_{a}(\operatorname{se}(Q) \unlhd b \unrhd s e(R)) \\
& =s e(Q) \unlhd b \unrhd s e(R) \\
& =s e(Q \triangleleft b \triangleright R) \\
& =s e\left(h_{a}(Q \triangleleft b \triangleright R)\right) .
\end{aligned}
$$

The second equality can be derived in a similar way, and this finishes the proof of (8).

We prove the lemma's statement by induction on $d(P)$. The base cases $P \in\{\mathrm{T}, \mathrm{F}\}$ follow immediately. Assume $P=Q \triangleleft a \triangleright R$, then

$$
\begin{array}{rlrl}
\operatorname{cr}(\operatorname{se}(Q \triangleleft a \triangleright R)) & =\operatorname{cr}(\operatorname{se}(Q) \unlhd a \unrhd \operatorname{se}(R)) & & \\
& =\operatorname{cr}\left(H_{a}(\operatorname{se}(Q))\right) \unlhd a \unrhd \operatorname{cr}\left(J_{a}(\operatorname{se}(R))\right) & & \\
& =\operatorname{cr}\left(\operatorname{se}\left(h_{a}(Q)\right)\right) \unlhd a \unrhd \operatorname{cr}\left(\operatorname{se}\left(j_{a}(R)\right)\right) & & \text { by (8) } \\
& =\operatorname{se}\left(\operatorname{cf}\left(h_{a}(Q)\right)\right) \unlhd a \unrhd \operatorname{se}\left(\operatorname{cf}\left(j_{a}(R)\right)\right) & & \text { by IH (and Lemma 4.3) } \\
& =\operatorname{se}\left(\operatorname{cf}\left(h_{a}(Q)\right) \triangleleft a \triangleright \operatorname{cf}\left(j_{a}(R)\right)\right) & & \\
& =\operatorname{se}(\operatorname{cf}(Q \triangleleft a \triangleright R)) .
\end{array}
$$

Finally, we relate conditional statements by means of their contractive evaluation trees.

Definition 4.13. Contractive valuation congruence, notation $=_{c s e}$, is defined on $C_{A}$ as follows:

$$
P={ }_{\text {cse }} Q \quad \Longleftrightarrow \quad \operatorname{cse}(P)=\operatorname{cse}(Q) .
$$

The following characterization result immediately implies that $=_{c s e}$ is a congruence relation on $C_{A}$ (and hence justifies calling it a congruence).

Proposition 4.14. For all $P, Q \in C_{A}, P={ }_{c s e} Q \Longleftrightarrow P={ }_{c b f} Q$. 
Proof. $(\Rightarrow)$ Assume $\operatorname{cse}(P)=\operatorname{cse}(Q)$, thus $\operatorname{cr}(\operatorname{se}(P))=\operatorname{cr}(\operatorname{se}(Q))$. By Corollary 2.19, $\operatorname{cr}(\operatorname{se}(b f(P)))=\operatorname{cr}(\operatorname{se}(b f(Q)))$, so by Lemma 4.12 $\operatorname{se}(\operatorname{cf}(b f(P)))=\operatorname{se}(c f(b f(Q)))$.

By Lemma 2.8 and auxiliary result (2) (see the proof of Lemma 3.5), it follows that $c f(b f(P))=$ $c f(b f(Q))$, that is, $P={ }_{c b f} Q$.

$(\Leftarrow)$ Assume $P={ }_{c b f} Q$, thus $c f(b f(P))=c f(b f(Q))$ and $\operatorname{se}(c f(b f(P)))=\operatorname{se}(c f(b f(Q)))$. By Lemma 4.12, $\operatorname{cr}(\operatorname{se}(b f(P)))=\operatorname{cr}(\operatorname{se}(b f(Q)))$. By Corollary 2.19, $\operatorname{se}(b f(P))=\operatorname{se}(P)$ and $\operatorname{se}(b f(Q))=\operatorname{se}(Q)$, so $\operatorname{cr}(\operatorname{se}(P))=\operatorname{cr}(\operatorname{se}(Q))$, that is, $P={ }_{c s e} Q$.

Our final result in this section is a completeness result for contractive valuation congruence.

Theorem 4.15 (Completeness of $\left.\mathrm{CP}_{c r}(A)\right)$. For all $P, Q \in C_{A}$,

$$
\mathrm{CP}_{c r}(A) \vdash P=Q \Longleftrightarrow P={ }_{\text {cse }} Q .
$$

Proof. Combine Theorem 4.10 and Proposition 4.14.

\section{Evaluation trees for memorizing valuation congruence}

In [4] we introduced $\mathrm{CP}_{m e m}$, memorizing $\mathrm{CP}$, as the extension of $\mathrm{CP}$ with the following axiom:

$$
x \triangleleft y \triangleright(z \triangleleft u \triangleright(v \triangleleft y \triangleright w))=x \triangleleft y \triangleright(z \triangleleft u \triangleright w) .
$$

(CPmem)

Axiom (CPmem expresses that the first evaluation value of $y$ is memorized. More precisely, a "memorizing evaluation" is one with the property that upon the evaluation of a compound propositional statement, the first evaluation value of each atom is memorized throughout the evaluation. We write $\mathrm{CP}_{m e m}$ for the set $\mathrm{CP} \cup\{(\overline{\mathrm{CPm} m})\}$ of axioms.

Replacing the variable $y$ in axiom (CPmem) by $\mathrm{F} \triangleleft y \triangleright T$ and/or the variable $u$ by $\mathrm{F} \triangleleft u \triangleright T$ yields all other memorizing patterns:

$$
\begin{aligned}
& (z \triangleleft u \triangleright(w \triangleleft y \triangleright v)) \triangleleft y \triangleright x=(z \triangleleft u \triangleright w) \triangleleft y \triangleright x, \\
& x \triangleleft y \triangleright((v \triangleleft y \triangleright w) \triangleleft u \triangleright z)=x \triangleleft y \triangleright(w \triangleleft u \triangleright z), \\
& ((w \triangleleft y \triangleright v) \triangleleft u \triangleright z) \triangleleft y \triangleright x=(w \triangleleft u \triangleright z) \triangleleft y \triangleright x .
\end{aligned}
$$

Hence, the duality priciple also holds in $\mathrm{CP}_{m e m}$. Furthermore, if we replace in axiom (CPmem) $u$ by $\mathrm{F}$, we find the contraction law

$$
x \triangleleft y \triangleright(v \triangleleft y \triangleright w)=x \triangleleft y \triangleright w,
$$

and replacing $y$ by $\mathrm{F} \triangleleft y \triangleright T$ then yields the dual contraction law

$$
(w \triangleleft y \triangleright v) \triangleleft y \triangleright x=w \triangleleft y \triangleright x .
$$

Hence, $\mathrm{CP}_{m e m}$ is an axiomatic extension of $\mathrm{CP}_{c r}(A)$.

We define a proper subset of basic forms with the property that each propositional statement can be proved equal to such a basic form.

Definition 5.1. Let $A^{\prime}$ be a subset of $A$. Mem-basic forms over $A^{\prime}$ are inductively defined:

- $\mathrm{T}$ and $\mathrm{F}$ are mem-basic forms over $A^{\prime}$, and

- $P \triangleleft a \triangleright Q$ is a mem-basic form over $A^{\prime}$ if $a \in A^{\prime}$ and $P$ and $Q$ are mem-basic forms over $A^{\prime} \backslash\{a\}$. 
$P$ is a mem-basic form if for some $A^{\prime} \subset A, P$ is a mem-basic form over $A^{\prime}$.

Note that if $A$ is finite, the number of mem-basic forms is also finite. It will turn out useful to define a function that transforms conditional statements into mem-basic forms.

Definition 5.2. The mem-basic form function mbf: $C_{A} \rightarrow C_{A}$ is defined by

$$
m b f(P)=m f(b f(P)) .
$$

The auxiliary function $m f: B F_{A} \rightarrow B F_{A}$ is defined as follows:

$$
\begin{aligned}
m f(\mathrm{~T}) & =\mathrm{T} \\
m f(\mathrm{~F}) & =\mathrm{F}, \\
m f(P \triangleleft a \triangleright Q) & =m f\left(\ell_{a}(P)\right) \triangleleft a \triangleright m f\left(r_{a}(Q)\right) .
\end{aligned}
$$

For $a \in A$, the auxiliary functions $\ell_{a}: B F_{A} \rightarrow B F_{A}$ and $r_{a}: B F_{A} \rightarrow B F_{A}$ are defined by

$$
\begin{aligned}
\ell_{a}(\mathrm{~T}) & =\mathrm{T}, \\
\ell_{a}(\mathrm{~F}) & =\mathrm{F}, \\
\ell_{a}(P \triangleleft b \triangleright Q) & = \begin{cases}\ell_{a}(P) & \text { if } b=a, \\
\ell_{a}(P) \triangleleft b \triangleright \ell_{a}(Q) & \text { otherwise, }\end{cases}
\end{aligned}
$$

and

$$
\begin{aligned}
r_{a}(\mathrm{~T}) & =\mathrm{T}, \\
r_{a}(\mathrm{~F}) & =\mathrm{F}, \\
r_{a}(P \triangleleft b \triangleright Q) & = \begin{cases}r_{a}(Q) & \text { if } b=a, \\
r_{a}(P) \triangleleft b \triangleright r_{a}(Q) & \text { otherwise. }\end{cases}
\end{aligned}
$$

Thus, $m b f$ maps a conditional statement $P$ to $b f(P)$ and then transforms $b f(P)$ according to the auxiliary functions $m f, \ell_{a}$, and $r_{a}$. We will use the following inequalities.

Lemma 5.3. For all $a \in A$ and $P \in B F_{A}, d(P) \geq d\left(\ell_{a}(P)\right)$ and $d(P) \geq d\left(r_{a}(P)\right)$.

Proof. Fix some $a \in A$. We prove these inequalities by structural induction on $P$. The base cases $P \in\{\mathrm{T}, \mathrm{F}\}$ are trivial. For the inductive case $P=Q \triangleleft b \triangleright R$ we have to distinguish the cases $b=a$ and $b \neq a$. If $b=a$, then

$$
\begin{aligned}
d(Q \triangleleft a \triangleright R) & =1+\max \{d(Q), d(R)\} \\
& \geq 1+d(Q) \\
& \geq 1+d\left(\ell_{a}(Q)\right) \\
& =1+d\left(\ell_{a}(Q \triangleleft a \triangleright R)\right),
\end{aligned}
$$

by $\mathrm{IH}$

and $d(Q \triangleleft a \triangleright R) \geq d\left(r_{a}(Q \triangleleft a \triangleright R)\right)$ follows in a similar way.

If $b \neq a$, then

$$
\begin{aligned}
d(Q \triangleleft b \triangleright R) & =1+\max \{d(Q), d(R)\} \\
& \geq 1+\max \left\{d\left(\ell_{a}(Q)\right), d\left(\ell_{a}(R)\right)\right\} \\
& =d\left(\ell_{a}(Q) \triangleleft b \triangleright \ell_{a}(R)\right) \\
& =d\left(\ell_{a}(Q \triangleleft b \triangleright R)\right),
\end{aligned}
$$

and $d(Q \triangleleft b \triangleright R) \geq d\left(r_{a}(Q \triangleleft b \triangleright R)\right)$ follows in a similar way. 
Lemma 5.4. For all $P \in C_{A}, m b f(P)$ is a mem-basic form.

Proof. We first prove an auxiliary result:

For all $P \in B F_{A}, m f(P)$ is a mem-basic form.

This follows by induction on the depth $d(P)$ of $P$. If $d(P)=0$, then $P \in\{\mathrm{T}, \mathrm{F}\}$, and hence $m f(P)=P$ is a mem-basic form. For the inductive case $d(P)=n+1$ it must be the case that $P=Q \triangleleft a \triangleright R$. We find

$$
m f(Q \triangleleft a \triangleright R)=m f\left(\ell_{a}(Q)\right) \triangleleft a \triangleright m f\left(r_{a}(R)\right),
$$

which is a mem-basic form because by Lemma 5.3, $\ell_{a}(Q)$ and $r_{a}(R)$ are basic forms with depth smaller than or equal to $n$, so by the induction hypothesis, $m f\left(\ell_{a}(Q)\right)$ is a mem-basic form over $A_{Q}$ and $m f\left(r_{a}(R)\right)$ is a mem-basic form over $A_{R}$ for suitable subsets $A_{Q}$ and $A_{R}$ of $A$. Notice that by definition of $\ell_{a}$ and $r_{a}$ we can assume that the atom $a$ does not occur in $A_{Q} \cup A_{R}$. Hence, $m f\left(\ell_{a}(Q)\right) \triangleleft a \triangleright m f\left(r_{a}(R)\right)$ is a mem-basic form over $A_{Q} \cup A_{R} \cup\{a\}$, which completes the proof of (11).

The lemma's statement now follows by structural induction: the base cases (comprising a single atom $a$ ) are again trivial, and for the inductive case,

$$
m b f(P \triangleleft Q \triangleright R)=m f(b f(P \triangleleft Q \triangleright R))=m f(S)
$$

for some basic form $S$ by Lemma 2.13, and by (11), $m f(S)$ is a mem-basic form.

With Lemma 5.4 we can easily prove the following result.

Proposition 5.5 ( $m b f$ is a normalization function). For each $P \in C_{A}, m b f(P)$ is a mem-basic form, and for each mem-basic form $P, m b f(P)=P$.

Proof. The first statement is Lemma 5.4. For the second statement, it suffices by Lemma 2.14 to prove that $m f(P)=P$. We prove this by induction on $d(P)$. The base cases $P \in\{\mathrm{T}, \mathrm{F}\}$ are trivial, and for the inductive case, assume $P=Q \triangleleft a \triangleright R$, thus $m f(P)=m f\left(\ell_{a}(Q)\right) \triangleleft a \triangleright m f\left(r_{a}(R)\right)$. Because $P$ is a mem-basic form, $Q$ and $R$ are mem-basic forms in which $a$ does not occur, and thus $\ell_{a}(Q)=Q$ and $r_{a}(R)=R$. By induction, $m f(Q)=Q$ and $m f(R)=R$, and thus $m f(P)=P$.

Lemma 5.6. For all $P \in B F_{A}, \mathrm{CP}_{m e m} \vdash P=m f(P)$.

Proof. We first prove an auxiliary result:

$$
\begin{aligned}
& \text { For all } a \in A \text { and } P, Q \in B F_{A}, \mathrm{CP}_{m e m} \vdash P \triangleleft a \triangleright Q=P \triangleleft a \triangleright r_{a}(Q) \text {, } \\
& P \triangleleft a \triangleright Q=\ell_{a}(P) \triangleleft a \triangleright Q \text {. }
\end{aligned}
$$

Fix some $a \in A$. We prove (12) by structural induction on $Q$. The base cases $Q \in\{\mathrm{T}, \mathrm{F}\}$ are trivial. For the inductive case $Q=Q_{1} \triangleleft b \triangleright Q_{2}$ we have to distinguish the cases $b=a$ and $b \neq a$. If $b=a$, then $r_{a}(Q)=r_{a}\left(Q_{2}\right)$ and

$$
\begin{aligned}
\mathrm{CP}_{m e m} \vdash P \triangleleft a \triangleright\left(Q_{1} \triangleleft a \triangleright Q_{2}\right) & =P \triangleleft a \triangleright Q_{2} & & \text { by (9) } \\
& =P \triangleleft a \triangleright r_{a}\left(Q_{2}\right) & & \text { by IH } \\
& =P \triangleleft a \triangleright r_{a}(Q) . & &
\end{aligned}
$$


If $b \neq a$, then $r_{a}(Q)=r_{a}\left(Q_{1}\right) \triangleleft b \triangleright r_{a}\left(Q_{2}\right)$ and

$$
\begin{aligned}
\mathrm{CP}_{\text {mem }} & \vdash P \triangleleft a \triangleright\left(Q_{1} \triangleleft b \triangleright Q_{2}\right) & & \\
& =P \triangleleft a \triangleright\left(\left(\mathrm{T} \triangleleft a \triangleright Q_{1}\right) \triangleleft b \triangleright\left(\mathrm{T} \triangleleft a \triangleright Q_{2}\right)\right) & & \text { by (CPmem), (CPm2) } \\
& =P \triangleleft a \triangleright\left(\left(\mathrm{T} \triangleleft a \triangleright r_{a}\left(Q_{1}\right)\right) \triangleleft b \triangleright\left(\mathrm{T} \triangleleft a \triangleright r_{a}\left(Q_{2}\right)\right)\right) & & \text { by IH (twice) } \\
& =P \triangleleft a \triangleright\left(r_{a}\left(Q_{1}\right) \triangleleft b \triangleright r_{a}\left(Q_{2}\right)\right) & & \text { by (CPmem), (CPm2) } \\
& =P \triangleleft a \triangleright r_{a}(Q) . & &
\end{aligned}
$$

Auxiliary result (13) follows in a similar way with help of axioms (CPm1) and (CPm3).

The lemma's statement follows by induction on $d(P)$. The base cases $P \in\{\mathrm{T}, \mathrm{F}\}$ are trivial. For the inductive case, assume $P=Q \triangleleft a \triangleright R$. We derive

$$
\begin{aligned}
\mathrm{CP}_{\text {mem }} \vdash Q \triangleleft a \triangleright R & =\ell_{a}(Q) \triangleleft a \triangleright r_{a}(R) & & \text { by (12), (13) } \\
& =m f\left(\ell_{a}(Q)\right) \triangleleft a \triangleright m f\left(r_{a}(R)\right) & & \text { by IH (and Lemma [5.3) } \\
& =m f(Q \triangleleft a \triangleright R) . & &
\end{aligned}
$$

Theorem 5.7. For all $P \in C_{A}, \mathrm{CP}_{m e m} \vdash P=m b f(P)$.

Proof. By Theorem 2.18 and Corollary 2.19, $\mathrm{CP}_{m e m} \vdash P=b f(P)$, and by Lemma 5.6, $\mathrm{CP}_{m e m} \vdash$ $b f(P)=m f(b f(P))$, and $m f(b f(P))=m b f(P)$.

Definition 5.8. The binary relation $={ }_{m b f}$ on $C_{A}$ is defined as follows:

$$
P={ }_{m b f} Q \Longleftrightarrow m b f(P)=\operatorname{mbf}(Q) .
$$

Theorem 5.9. For all $P, Q \in C_{A}, \mathrm{CP}_{m e m} \vdash P=Q \Longleftrightarrow P={ }_{m b f} Q$.

Proof. $(\Rightarrow)$ Assume $\mathrm{CP}_{\text {mem }} \vdash P=Q$. Then, by Theorem 5.7. $\mathrm{CP}_{\text {mem }} \vdash m b f(P)=m b f(Q)$. In 4 the following two statements are proved (Theorem 8.1 and Lemma 8.4), where $={ }_{m e m}$ is a binary relation on $C_{A}$ :

1. For all $P, Q \in C_{A}, \quad \mathrm{CP}_{m e m} \vdash P=Q \Longleftrightarrow P={ }_{m e m} Q$.

2. For all mem-basic forms $P$ and $Q, \quad P={ }_{m e m} Q \Rightarrow P=Q$.

By Lemma 5.4 these statements imply $m b f(P)=m b f(Q)$, that is, $P={ }_{m b f} Q$.

$(\Leftarrow)$ Assume $P={ }_{m b f} Q$. By Theorem 5.7. $\mathrm{CP}_{m e m} \vdash P=Q$.

Hence, the relation $=_{m b f}$ is axiomatized by $\mathrm{CP}_{m e m}$ and is thus a congruence. We define a transformation on evaluation trees that mimics the function $m b f$, and prove that equality of two such transformed trees characterizes the congruence that is axiomatized by $\mathrm{CP}_{m e m}$.

Definition 5.10. The unary memorizing evaluation function

$$
\text { mse }: C_{A} \rightarrow \mathcal{T}_{A}
$$

yields memorizing evaluation trees and is defined by

$$
m s e(P)=m(s e(P)) \text {. }
$$


The auxiliary function $m: \mathcal{T}_{A} \rightarrow \mathcal{T}_{A}$ is defined as follows $(a \in A)$ :

$$
\begin{aligned}
m(\mathrm{~T}) & =\mathrm{T}, \\
m(\mathrm{~F}) & =\mathrm{F}, \\
m(X \unlhd a \unrhd Y) & =m\left(L_{a}(X)\right) \unlhd a \unrhd m\left(R_{a}(Y)\right) .
\end{aligned}
$$

For $a \in A$, the auxiliary functions $L_{a}: \mathcal{T}_{A} \rightarrow \mathcal{T}_{A}$ and $R_{a}: \mathcal{T}_{A} \rightarrow \mathcal{T}_{A}$ are defined by

$$
\begin{aligned}
L_{a}(\mathrm{~T}) & =\mathrm{T}, \\
L_{a}(\mathrm{~F}) & =\mathrm{F}, \\
L_{a}(X \unlhd b \unrhd Y) & = \begin{cases}L_{a}(X) & \text { if } b=a, \\
L_{a}(X) \unlhd b \unrhd L_{a}(Y) & \text { otherwise, }\end{cases}
\end{aligned}
$$

and

$$
\begin{aligned}
R_{a}(\mathrm{~T}) & =\mathrm{T}, \\
R_{a}(\mathrm{~F}) & =\mathrm{F}, \\
R_{a}(X \unlhd b \unrhd Y) & = \begin{cases}R_{a}(Y) & \text { if } b=a, \\
R_{a}(X) \unlhd b \unrhd R_{a}(Y) & \text { otherwise. }\end{cases}
\end{aligned}
$$

As a simple example we depict $\operatorname{se}((a \triangleleft b \triangleright \mathrm{F}) \triangleleft a \triangleright \mathrm{F})$ and the memorizing evaluation tree $m s e((a \triangleleft b \triangleright \mathrm{F}) \triangleleft a \triangleright \mathrm{F})$ :
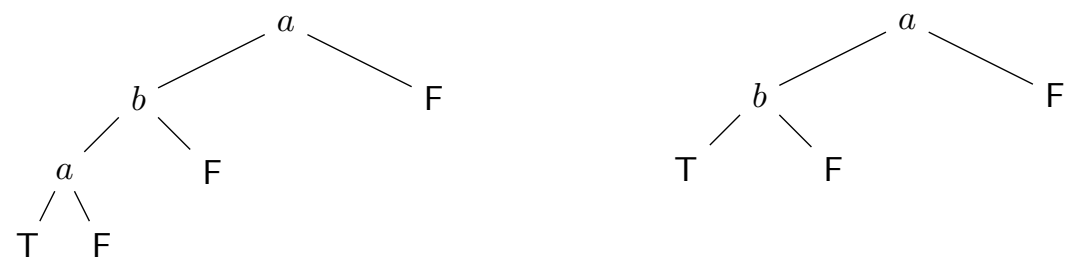

The similarities between mse and the function $m b f$ will of course be exploited.

Lemma 5.11. For all $P \in B F_{A}, m(s e(P))=s e(m f(P))$.

Proof. We first prove an auxiliary result:

For all $a \in A$ and $P \in B F_{A}, L_{a}(s e(P))=s e\left(\ell_{a}(P)\right)$ and $R_{a}(s e(P))=s e\left(r_{a}(P)\right)$.

Fix some $a \in A$. We prove (14) by structural induction on $P$. The base cases $P \in\{\mathrm{T}, \mathrm{F}\}$ are trivial. For the inductive case $P=Q \triangleleft b \triangleright R$ we have to distinguish the cases $b=a$ and $b \neq a$. If $b=a$, then

$$
\begin{aligned}
L_{a}(\operatorname{se}(Q \triangleleft a \triangleright R)) & =L_{a}(\operatorname{se}(Q) \unlhd a \unrhd s e(R)) \\
& =L_{a}(\operatorname{se}(Q)) \\
& =\operatorname{se}\left(\ell_{a}(Q)\right) \\
& =\operatorname{se}\left(\ell_{a}(Q \triangleleft a \triangleright R)\right),
\end{aligned}
$$

by IH 
and if $b \neq a$, then

$$
\begin{aligned}
L_{a}(\operatorname{se}(Q \triangleleft b \triangleright R)) & =L_{a}(\operatorname{se}(Q) \unlhd b \unrhd \operatorname{se}(R)) & \\
& =L_{a}(\operatorname{se}(Q)) \unlhd b \unrhd L_{a}(\operatorname{se}(R)) & \\
& =\operatorname{se}\left(\ell_{a}(Q)\right) \triangleleft b \triangleright \operatorname{se}\left(\ell_{a}(R)\right) & \text { by IH } \\
& =\operatorname{se}\left(\ell_{a}(Q \triangleleft b \triangleright R)\right) . &
\end{aligned}
$$

The second equality can be derived in a similar way, and this finishes the proof of (14).

We prove the lemma's statement by induction on $d(P)$. The base cases $P \in\{\mathrm{T}, \mathrm{F}\}$ follow immediately. Assume $P=Q \triangleleft a \triangleright R$, then

$$
\begin{array}{rlrl}
m(\operatorname{se}(Q \triangleleft a \triangleright R)) & =m(\operatorname{se}(Q) \unlhd a \unrhd s e(R)) & & \\
& =m\left(L_{a}(\operatorname{se}(Q))\right) \unlhd a \unrhd m\left(R_{a}(\operatorname{se}(R))\right) & & \\
& =m\left(\operatorname{se}\left(\ell_{a}(Q)\right)\right) \unlhd a \unrhd m\left(\operatorname{se}\left(r_{a}(R)\right)\right) & & \text { by (14) } \\
& =\operatorname{se}\left(m f\left(\ell_{a}(Q)\right)\right) \unlhd a \unrhd \operatorname{se}\left(m f\left(r_{a}(R)\right)\right) & & \text { by IH (and Lemma 5.3) } \\
& =\operatorname{se}\left(m f\left(\ell_{a}(Q)\right) \triangleleft a \triangleright m f\left(r_{a}(R)\right)\right) & & \\
& =s e(m f(Q \triangleleft a \triangleright R)) .
\end{array}
$$

Definition 5.12. Memorizing valuation congruence, notation $=_{m s e}$, is defined on $C_{A}$ as follows:

$$
P={ }_{m s e} Q \quad \Longleftrightarrow \quad m s e(P)=m s e(Q)
$$

The following characterization result immediately implies that $=_{m s e}$ is indeed a congruence relation on $C_{A}$.

Proposition 5.13. For all $P, Q \in C_{A}, P={ }_{m s e} Q \Longleftrightarrow P={ }_{m b f} Q$.

Proof. $(\Rightarrow)$ Assume $m s e(P)=m s e(Q)$, thus $m(\operatorname{se}(P))=m(s e(Q))$. By Corollary 2.19. $m(\operatorname{se}(b f(P)))=m(\operatorname{se}(b f(Q)))$, so by Lemma 5.11 .

$$
s e(m f(b f(P)))=s e(m f(b f(Q))) .
$$

By Lemma 2.8, it follows that $m f(b f(P))=m f(b f(Q))$, that is, $P={ }_{m b f} Q$.

$(\Leftarrow)$ If $P={ }_{m b f} Q$, then $s e(m f(b f(P)))=\operatorname{se}(m f(b f(Q)))$, and by Lemma 5.11

$$
m(\operatorname{se}(b f(P)))=m(\operatorname{se}(b f(Q))) .
$$

By Corollary 2.19, $m(\operatorname{se}(P))=m(\operatorname{se}(Q))$, that is, $P={ }_{m s e} Q$.

We end this section with a completeness result for memorizing valuation congruence.

Theorem 5.14 (Completeness of $\mathrm{CP}_{m e m}$ ). For all $P, Q \in C_{A}$,

$$
\mathrm{CP}_{m e m} \vdash P=Q \Longleftrightarrow P={ }_{m s e} Q .
$$

Proof. Combine Theorem 5.9 and Proposition 5.13 


\section{Evaluation trees for static valuation congruence}

The most identifying axiomatic extension of CP we consider can be defined by adding the following axiom to $\mathrm{CP}_{m e m}$ :

$$
\mathrm{F} \triangleleft x \triangleright \mathrm{F}=\mathrm{F} .
$$

So, the evaluation value of each atom in a conditional statement is memorized, and by axiom (CPs), no atom $a$ can have a side effect because $\mathrm{T} \triangleleft(\mathrm{F} \triangleleft a \triangleright \mathrm{F}) \triangleright P=\mathrm{T} \triangleleft \mathrm{F} \triangleright P=P$ for all $P \in C_{A}$. We write $\mathrm{CP}_{s}$ for the set of these axioms, thus

$$
\mathrm{CP}_{s}=\mathrm{CP}_{m e m} \cup\{\underline{\underline{\mathrm{CPS}}}\}=\mathrm{CP} \cup\{\underline{\underline{\mathrm{CPmem}}}, \underline{\underline{\mathrm{CPS}}}\} \text {. }
$$

Observe that $\mathrm{CP}_{s} \vdash \mathrm{T}=\mathrm{T} \triangleleft(\mathrm{F} \triangleleft x \triangleright \mathrm{F}) \triangleright \mathrm{T}=(\mathrm{T} \triangleleft \mathrm{F} \triangleright \mathrm{T}) \triangleleft x \triangleright(\mathrm{T} \triangleleft \mathrm{F} \triangleright \mathrm{T})=\mathrm{T} \triangleleft x \triangleright \mathrm{T}$, so the duality principle holds in $\mathrm{CP}_{s}$. The following lemma is a direct consequence of axiom (CPS).

Lemma 6.1. For all $P, Q \in C_{A}, \mathrm{CP}_{s} \vdash P=P \triangleleft Q \triangleright P$.

Proof.

$$
\begin{aligned}
\mathrm{CP}_{s} \vdash P & =\mathrm{T} \triangleleft(\mathrm{F} \triangleleft Q \triangleright \mathrm{F}) \triangleright P & & \text { by (CPS), (CP2) } \\
& =(\mathrm{T} \triangleleft \mathrm{F} \triangleright P) \triangleleft Q \triangleright(\mathrm{T} \triangleleft \mathrm{F} \triangleright P) & & \text { by (CP4) } \\
& =P \triangleleft Q \triangleright P . & & \text { by (CP2 }
\end{aligned}
$$

Recall that the contraction laws (9), that is $x \triangleleft y \triangleright(v \triangleleft y \triangleright w)=x \triangleleft y \triangleright w$, and (10), that is $(w \triangleleft y \triangleright v) \triangleleft y \triangleright x=w \triangleleft y \triangleright x$, are derivable from $\mathrm{CP}_{m e m}$. A simple example on $\mathrm{CP}_{s}$ illustrates how the order of evaluation of $x$ and $y$ in $x \triangleleft y \triangleright \mathrm{F}$ can be swapped:

$$
x \triangleleft y \triangleright \mathrm{F}=y \triangleleft x \triangleright \mathrm{F} .
$$

Equation (15) can be derived as follows:

$$
\begin{aligned}
\mathrm{CP}_{s} \vdash x \triangleleft y \triangleright \mathrm{F} & =((\mathrm{T} \triangleleft x \triangleright \mathrm{F}) \triangleleft y \triangleright \mathrm{F}) \triangleleft x \triangleright((\mathrm{T} \triangleleft x \triangleright \mathrm{F}) \triangleleft y \triangleright \mathrm{F}) & & \text { by (CP3), Lemma 6.1 } \\
& =(\mathrm{T} \triangleleft y \triangleright \mathrm{F}) \triangleleft x \triangleright(\mathrm{F} \triangleleft y \triangleright \mathrm{F}) & & \text { by (9) and (10) } \\
& =y \triangleleft x \triangleright \mathrm{F} . & & \text { by (CP3), (CPS) }
\end{aligned}
$$

In [4] we defined $\mathrm{CP}_{s t}$ as the extension of $\mathrm{CP}$ with the following two axioms:

$$
\begin{aligned}
& (x \triangleleft y \triangleright z) \triangleleft u \triangleright v=(x \triangleleft u \triangleright v) \triangleleft y \triangleright(z \triangleleft u \triangleright v), \\
& (x \triangleleft y \triangleright z) \triangleleft y \triangleright u=x \triangleleft y \triangleright u .
\end{aligned}
$$

(the contraction law (10))

Axiom CPstat expresses how the order of evaluation of $u$ and $y$ can be swapped. Because we will rely on results for $\mathrm{CP}_{s t}$ recorded in [4], we first prove the following result.

Proposition 6.2. The axiom sets $\mathrm{CP}_{\text {st }}$ and $\mathrm{CP}_{s}$ are equally strong.

Proof. We show that all axioms in the one set are derivable from the other set. We first prove that the axiom (CPmem) is derivable from $\mathrm{CP}_{s t}$ :

$$
\begin{aligned}
\mathrm{CP}_{s t} & \vdash x \triangleleft y \triangleright(z \triangleleft u \triangleright(v \triangleleft y \triangleright w)) & & \\
& =x \triangleleft y \triangleright((v \triangleleft y \triangleright w) \triangleleft(\mathrm{F} \triangleleft u \triangleright \mathrm{T}) \triangleright z) & & \text { by (CP4), (CP2), (CP1) } \\
& =x \triangleleft y \triangleright((v \triangleleft(\mathrm{F} \triangleleft u \triangleright \mathrm{T}) \triangleright z) \triangleleft y \triangleright(w \triangleleft(\mathrm{F} \triangleleft u \triangleright \mathrm{T}) \triangleright z)) & & \text { by (CPstat) } \\
& =x \triangleleft y \triangleright(w \triangleleft(\mathrm{F} \triangleleft u \triangleright \mathrm{T}) \triangleright z) & & \text { by (9) } \\
& =x \triangleleft y \triangleright(z \triangleleft u \triangleright w), & & \text { by (CP4), (CP2), (CP1) }
\end{aligned}
$$


where the contraction law (9) is derivable from $\mathrm{CP}_{s t}$ : replace $y$ by $\mathrm{F} \triangleleft y \triangleright T$ in (10). Hence $\mathrm{CP}_{s} \vdash$ (CPmem). If $u=v=\mathrm{F}$ in axiom (CPstat), we find $\mathrm{F} \triangleleft x \triangleright \mathrm{F}=\mathrm{F}$, hence $\mathrm{CP}_{s t} \vdash \mathrm{CP}_{s}$.

In order to show that $\mathrm{CP}_{s} \vdash \mathrm{CP}_{s t}$ we have to derive $\mathrm{CP}_{s} \vdash$ (CPstat):

$$
\begin{aligned}
\mathrm{CP}_{s} \vdash(x \triangleleft y \triangleright z) \triangleleft u \triangleright v & =(x \triangleleft y \triangleright(z \triangleleft u \triangleright v)) \triangleleft u \triangleright v & & \text { by (CPm1) } \\
& =(x \triangleleft y \triangleright(z \triangleleft u \triangleright v)) \triangleleft u \triangleright(z \triangleleft u \triangleright v) & & \text { by (19) } \\
& =x \triangleleft(y \triangleleft u \triangleright \mathrm{F}) \triangleright(z \triangleleft u \triangleright v) & & \text { by (CP4), (CP2) } \\
& =x \triangleleft(u \triangleleft y \triangleright \mathrm{F}) \triangleright(z \triangleleft u \triangleright v) & & \text { by (15) } \\
& =(x \triangleleft u \triangleright(z \triangleleft u \triangleright v)) \triangleleft y \triangleright(z \triangleleft u \triangleright v) & & \text { by (CP4), (CP2) } \\
& =(x \triangleleft u \triangleright v) \triangleleft y \triangleright(z \triangleleft u \triangleright v) . & & \text { by (19) }
\end{aligned}
$$

Given a finite, ordered subset of atoms we define a proper subset of basic forms with the property that each propositional statement over these atoms can be proved equal to such a basic form.

Definition 6.3. Let $A^{u} \subset A^{*}$ be the set of strings over $A$ with the property that each $\sigma \in A^{u}$ contains no multiple occurrences of the same atom 4 St-basic forms over $\sigma \in A^{u}$ are defined as follows:

- $\mathrm{T}$ and $\mathrm{F}$ are st-basic forms over $\epsilon$, and

- $P \triangleleft a \triangleright Q$ is an st-basic form over $\rho a \in A^{u}$ if $P$ and $Q$ are st-basic forms over $\rho$.

$P$ is an st-basic form if for some $\sigma \in A^{u}, P$ is an st-basic form over $\sigma$.

For example, an st-basic form over $a b \in A^{u}$ has the following form:

$$
\left(B_{1} \triangleleft a \triangleright B_{2}\right) \triangleleft b \triangleright\left(B_{3} \triangleleft a \triangleright B_{4}\right)
$$

with $B_{i} \in\{\mathrm{T}, \mathrm{F}\}$. For $\sigma=a_{1} a_{2} \cdots a_{n} \in A^{u}$, there exist $2^{2^{n}}$ different st-basic forms over $\sigma$.

It will turn out useful to define a function that transforms conditional statements to st-basic forms. Therefore, given $\sigma \in A^{u}$ we consider terms in $C_{A^{\prime}}$, where $A^{\prime}$ is the finite subset of $A$ that contains the elements of $\sigma$.

Definition 6.4. The alphabet function $\alpha: A^{u} \rightarrow 2^{A}$ returns the set of atoms of a string in $A^{u}$ :

$$
\alpha(\epsilon)=\emptyset, \quad \text { and } \quad \alpha(\sigma a)=\alpha(\sigma) \cup\{a\} .
$$

Definition 6.5. Let $\sigma \in A^{u}$. The conditional statement $E^{\sigma} \in B F_{\alpha(\sigma)}$ is defined as

$$
E^{\epsilon}=\mathrm{F} \quad \text { and, if } \sigma=\rho a, \quad E^{\sigma}=E^{\rho} \triangleleft a \triangleright E^{\rho} .
$$

So, for each $\sigma \in A^{u}, E^{\sigma}$ is an st-basic form over $\sigma$ in which the constant T does not occur, e.g.,

$$
E^{a b}=(\mathrm{F} \triangleleft a \triangleright \mathrm{F}) \triangleleft b \triangleright(\mathrm{F} \triangleleft a \triangleright \mathrm{F}) .
$$

\footnotetext{
${ }^{4}$ Recall that we write $\epsilon$ for the empty string, thus $\epsilon \in A^{u}$. If $A$ is finite, say $|A|=n$, then $\left|A^{u}\right|=a(n)$, where $a(0)=1$ and $a(k+1)=1+(k+1) \cdot a(k)$.
} 
Definition 6.6. The st-basic form function $s b f_{\sigma}: C_{\alpha(\sigma)} \rightarrow C_{\alpha(\sigma)}$ is defined by

$$
\operatorname{sbf}_{\sigma}(P)=m b f\left(\mathrm{~T} \triangleleft E^{\sigma} \triangleright P\right),
$$

where mbf is defined in Definition 5.2.

For example, $s b f_{a b}(a)=(\mathrm{T} \triangleleft a \triangleright \mathrm{F}) \triangleleft b \triangleright(\mathrm{T} \triangleleft a \triangleright \mathrm{F})$ and $s b f_{b a}(a)=(\mathrm{T} \triangleleft b \triangleright \mathrm{T}) \triangleleft a \triangleright(\mathrm{F} \triangleleft b \triangleright \mathrm{F})$.

The reason that $\operatorname{sse}_{\sigma}(P)$ is defined relative to some $\sigma \in A^{u}$ that covers the alphabet of $P$ is that in order to prove completeness of $\mathrm{CP}_{s}$ (and $\mathrm{CP}_{s t}$ ), we need to be able to relate conditional statements that contain different sets of atoms, but have equal st-basic forms for all appropriate $\sigma$, such as

$$
s b f_{b a}(\mathrm{~F})=s b f_{b a}(\mathrm{~F} \triangleleft a \triangleright \mathrm{F})=s b f_{b a}(\mathrm{~F} \triangleleft b \triangleright \mathrm{F})=(\mathrm{F} \triangleleft b \triangleright \mathrm{F}) \triangleleft a \triangleright(\mathrm{F} \triangleleft b \triangleright \mathrm{F}) .
$$

Lemma 6.7. Let $\sigma \in A^{u}$. For all $P \in C_{A}, \mathrm{CP}_{s} \vdash P=\mathrm{T} \triangleleft E^{\sigma} \triangleright P$.

Proof. By induction on the structure of $\sigma$. If $\sigma=\epsilon$, then $E^{\sigma}=\mathrm{F}$ and by axiom (CP2), $\mathrm{CP}_{s} \vdash$ $P=\mathrm{\top} \triangleleft E^{\epsilon} \triangleright P$. If $\sigma=\rho a$ for some $\rho \in A^{u}$ and $a \in A$, then $E^{\sigma}=E^{\rho} \triangleleft a \triangleright E^{\rho}$, and hence

$$
\begin{aligned}
\mathrm{CP}_{s} \vdash P & =P \triangleleft a \triangleright P & & \text { by Lemma 6.1 } \\
& =\left(\mathrm{\top} \triangleleft E^{\rho} \triangleright P\right) \triangleleft a \triangleright\left(\mathrm{\top} \triangleleft E^{\rho} \triangleright P\right) & & \text { by IH } \\
& =\mathrm{\top} \triangleleft\left(E^{\rho} \triangleleft a \triangleright E^{\rho}\right) \triangleright P . & & \text { by (CP4) }
\end{aligned}
$$

Lemma 6.8. Let $\sigma \in A^{u}$. For all $P \in C_{\alpha(\sigma)}$, sbf $f_{\sigma}(P)$ is an st-basic form.

Proof. We first prove an auxiliary result:

$$
\begin{aligned}
& \text { For all } a \in A \text { and } P, Q \in B F_{A}, \ell_{a}(P[\mathrm{~F} \mapsto Q])=\left(\ell_{a}(P)\right)\left[\mathrm{F} \mapsto \ell_{a}(Q)\right] \\
& \text { and } r_{a}(P[\mathrm{~F} \mapsto Q])=\left(r_{a}(P)\right)\left[\mathrm{F} \mapsto r_{a}(Q)\right]
\end{aligned}
$$

Both equalities follow easily by induction on the structure of $P$ and we only show the inductive case for the first one. Choose $a \in A$. If $P=P_{1} \triangleleft a \triangleright P_{2}$, then $\ell_{a}(P)=\ell_{a}\left(P_{1}\right)$ and

$$
\begin{aligned}
& \ell_{a}(P[\mathrm{~F} \mapsto Q])=\ell_{a}\left(P_{1}[\mathrm{~F} \mapsto Q] \triangleleft a \triangleright P_{2}[\mathrm{~F} \mapsto Q]\right)=\ell_{a}\left(P_{1}[\mathrm{~F} \mapsto Q]\right) \\
& \stackrel{\mathrm{IH}}{=}\left(\ell_{a}\left(P_{1}\right)\right)\left[\mathrm{F} \mapsto \ell_{a}(Q)\right]=\left(\ell_{a}(P)\right)\left[\mathrm{F} \mapsto \ell_{a}(Q)\right],
\end{aligned}
$$

and if $P=P_{1} \triangleleft b \triangleright P_{2}$ with $b \neq a$, then $\ell_{a}(P)=\ell_{a}\left(P_{1}\right) \triangleleft b \triangleright \ell_{a}\left(P_{2}\right)$ and

$$
\begin{aligned}
& \ell_{a}(P[\mathrm{~F} \mapsto Q])=\ell_{a}\left(P_{1}[\mathrm{~F} \mapsto Q] \triangleleft b \triangleright P_{2}[\mathrm{~F} \mapsto Q]\right)=\ell_{a}\left(P_{1}[\mathrm{~F} \mapsto Q]\right) \triangleleft b \triangleright \ell_{a}\left(P_{1}[\mathrm{~F} \mapsto Q]\right) \\
& \stackrel{\mathrm{IH}}{=}\left(\ell_{a}\left(P_{1}\right)\right)\left[\mathrm{F} \mapsto \ell_{a}(Q)\right] \triangleleft b \triangleright\left(\ell_{a}\left(P_{2}\right)\right)\left[\mathrm{F} \mapsto \ell_{a}(Q)\right]=\left(\ell_{a}(P)\right)\left[\mathrm{F} \mapsto \ell_{a}(Q)\right] .
\end{aligned}
$$

We prove the lemma's statement by induction on the structure of $\sigma$. If $\sigma=\epsilon$, then each $P \in C_{\alpha(\sigma)}$ contains no atoms. Hence, $b f(P) \in\{\mathrm{T}, \mathrm{F}\}$. If $b f(P)=\mathrm{T}$ then

$$
s b f_{\epsilon}(P)=m b f(\mathrm{~T} \triangleleft \mathrm{F} \triangleright P)=m f(b f(\mathrm{~T} \triangleleft \mathrm{F} \triangleright P))=m f(b f(P))=\mathrm{T},
$$

which is an st-basic form over $\epsilon$. The case for $b f(P)=\mathrm{F}$ is similar. 
If $\sigma=\rho a$ for some $\rho \in A^{u}$ and $a \in A$, then for each $P \in C_{\alpha(\sigma)}$,

$$
\begin{aligned}
& s b f_{\sigma}(P) \\
& =m b f\left(\mathrm{~T} \triangleleft E^{\sigma} \triangleright P\right) \\
& =m f\left(b f\left(\mathrm{~T} \triangleleft E^{\sigma} \triangleright P\right)\right) \\
& =m f\left(E^{\sigma}[\mathrm{F} \mapsto b f(P)]\right) \\
& =m f\left(E^{\rho}[\mathrm{F} \mapsto b f(P)] \triangleleft a \triangleright E^{\rho}[\mathrm{F} \mapsto b f(P)]\right) \\
& =m f\left(\ell_{a}\left(E^{\rho}[\mathrm{F} \mapsto b f(P)]\right)\right) \triangleleft a \triangleright m f\left(r_{a}\left(E^{\rho}[\mathrm{F} \mapsto b f(P)]\right)\right) \\
& =m f\left(\ell_{a}\left(E^{\rho}\right)\left[\mathrm{F} \mapsto \ell_{a}(b f(P))\right]\right) \triangleleft a \triangleright m f\left(r_{a}\left(E^{\rho}\right)\left[\mathrm{F} \mapsto r_{a}(b f(P))\right]\right) \quad \text { by (16) } \\
& =m f\left(E^{\rho}\left[\mathrm{F} \mapsto \ell_{a}(b f(P))\right]\right) \triangleleft a \triangleright m f\left(E^{\rho}\left[\mathrm{F} \mapsto r_{a}(b f(P))\right]\right) \\
& =m f\left(E^{\rho}\left[\mathrm{F} \mapsto b f\left(\ell_{a}(b f(P))\right)\right]\right) \triangleleft a \triangleright m f\left(E^{\rho}\left[\mathrm{F} \mapsto b f\left(r_{a}(b f(P))\right)\right]\right) \\
& =m f\left(b f\left(\mathrm{~T} \triangleleft E^{\rho} \triangleright \ell_{a}(b f(P))\right)\right) \triangleleft a \triangleright m f\left(b f\left(\mathrm{~T} \triangleleft E^{\rho} \triangleright r_{a}(b f(P))\right)\right) \\
& =s b f_{\rho}\left(\ell_{a}(b f(P))\right) \triangleleft a \triangleright s b f_{\rho}\left(r_{a}(b f(P))\right), \quad \text { by } \mathrm{IH}
\end{aligned}
$$

where (17) follows because $\ell_{a}(b f(P))$ and $r_{a}(b f(P))$ are conditional statements in $C_{\alpha(\rho)}$ (thus, not containing $a)$, so by induction, $s b f_{\rho}\left(\ell_{a}(b f(P))\right)$ and $s b f_{\rho}\left(r_{a}(b f(P))\right)$ are st-basic forms over $\rho$. Hence, $s b f_{\sigma}(P)$ is an st-basic form over $\sigma$.

With Lemma 6.8 we can easily prove the following result.

Proposition 6.9 ( $s b f_{\sigma}$ is a normalization function). Let $\sigma \in A^{u}$. For each $P \in C_{\alpha(\sigma)}, s b f_{\sigma}(P)$ is an st-basic form over $\sigma$, and for each st-basic form $P$ over $\sigma, \operatorname{sbf}_{\sigma}(P)=P$.

Proof. The first statement is Lemma 6.8. We prove the second statement by induction on the structure of $\sigma$. If $\sigma=\epsilon, s b f_{\sigma}(P)=P$ by definition.

If $\sigma=\rho a$, then $P=P_{1} \triangleleft a \triangleright P_{2}$ with $P_{i}$ st-basic forms over $\rho$, thus $\ell_{a}\left(P_{1}\right)=P_{1}$ and $r_{a}\left(P_{2}\right)=$ $P_{2}$. For brevity, we identify below $b f(Q)$ and $Q$ for all $Q \in B F_{A}$ :

$$
\begin{aligned}
s b f_{\sigma}(P) & =m b f\left(\mathrm{~T} \triangleleft E^{\rho a} \triangleright\left(P_{1} \triangleleft a \triangleright P_{2}\right)\right) & & \\
& =m f\left(E^{\rho a}\left[\mathrm{~F} \mapsto P_{1} \triangleleft a \triangleright P_{2}\right]\right) & & \text { as above } \\
& =m f\left(E^{\rho}\left[\mathrm{F} \mapsto P_{1} \triangleleft a \triangleright P_{2}\right] \triangleleft a \triangleright E^{\rho}\left[\mathrm{F} \mapsto P_{1} \triangleleft a \triangleright P_{2}\right]\right) & & \\
& =m f\left(\ell_{a}\left(E^{\rho}\left[\mathrm{F} \mapsto P_{1} \triangleleft a \triangleright P_{2}\right]\right) \triangleleft a \triangleright m f\left(r_{a}\left(E^{\rho}\left[\mathrm{F} \mapsto P_{1} \triangleleft a \triangleright P_{2}\right]\right)\right)\right. & & \\
& =m f\left(\ell_{a}\left(E^{\rho}\right)\left[\mathrm{F} \mapsto \ell_{a}\left(P_{1} \triangleleft a \triangleright P_{2}\right]\right)\right) \triangleleft a \triangleright m f\left(r_{a}\left(E^{\rho}\right)\left[\mathrm{F} \mapsto r_{a}\left(P_{1} \triangleleft a \triangleright P_{2}\right]\right)\right) & & \text { by }(\underline{16}) \\
& =m f\left(E^{\rho}\left[\mathrm{F} \mapsto P_{1}\right]\right) \triangleleft a \triangleright m f\left(E^{\rho}\left[\mathrm{F} \mapsto P_{2}\right]\right) & & \text { by } a \notin \alpha(\rho) \\
& =s b f_{\rho}\left(P_{1}\right) \triangleleft a \triangleright s b f_{\rho}\left(P_{2}\right) & & \\
& =P_{1} \triangleleft a \triangleright P_{2} . & & \text { by IH }
\end{aligned}
$$

Lemma 6.10. Let $\sigma \in A^{u}$. For all $P \in C_{\alpha(\sigma)}, \mathrm{CP}_{s} \vdash P=s b f_{\sigma}(P)$.

Proof. By Lemma 6.7 $\mathrm{CP}_{s} \vdash P=\mathrm{T} \triangleleft E^{\sigma} \triangleright P$. By Theorem 5.7 $\mathrm{CP}_{s} \vdash \mathrm{T} \triangleleft E^{\sigma} \triangleright P=$ $m b f\left(\mathrm{~T} \triangleleft E^{\sigma} \triangleright P\right)$, hence $\mathrm{CP}_{s} \vdash P=s b f_{\sigma}(P)$.

Definition 6.11. Let $\sigma \in A^{u}$. The binary relation $=_{s b f, \sigma}$ on $C_{\alpha(\sigma)}$ is defined as follows:

$$
P={ }_{s b f, \sigma} Q \Longleftrightarrow s b f_{\sigma}(P)=s b f_{\sigma}(Q) .
$$


Theorem 6.12. Let $\sigma \in A^{u}$. For all $P, Q \in C_{\alpha(\sigma)}, \mathrm{CP}_{s} \vdash P=Q \Longleftrightarrow P={ }_{s b f, \sigma} Q$.

Proof. $(\Rightarrow)$ Assume $\mathrm{CP}_{s} \vdash P=Q$. Then, by Lemma 6.10, $\mathrm{CP}_{s} \vdash s b f_{\sigma}(P)=s b f_{\sigma}(Q)$, and by Proposition 6.2. $\mathrm{CP}_{s t} \vdash s b f_{\sigma}(P)=s b f_{\sigma}(Q)$. In 4 the following two statements are proved (Theorem 9.1 and an auxiliary result in its proof), where $=_{s t}$ is a binary relation on $C_{A}$ :

1. For all $P, Q \in C_{A}, \quad \mathrm{CP}_{s t} \vdash P=Q \Longleftrightarrow P={ }_{s t} Q$.

2. For all st-basic forms $P$ and $Q, \quad P={ }_{s t} Q \Rightarrow P=Q$.

By Lemma 6.8 these statements imply $s b f_{\sigma}(P)=s b f_{\sigma}(Q)$, and thus $P={ }_{s b f, \sigma} Q$.

$(\Leftarrow)$ Assume $P={ }_{s b f, \sigma} Q$, thus $\mathrm{T} \triangleleft E^{\sigma} \triangleright P={ }_{m b f} \mathrm{~T} \triangleleft E^{\sigma} \triangleright Q$. By Theorem 5.9 $\mathrm{CP}_{m e m} \vdash$ $\mathrm{T} \triangleleft E^{\sigma} \triangleright P=\mathrm{T} \triangleleft E^{\sigma} \triangleright Q$, and by Lemma 6.7 this implies $\mathrm{CP}_{s} \vdash P=Q$.

Hence, the relation $={ }_{s b f, \sigma}$ is a congruence on $C_{\alpha(\sigma)}$ that is axiomatized by $\mathrm{CP}_{s}$. We define a transformation on evaluation trees that mimics the function $s b f_{\sigma}$ and prove that equality of two such transformed trees characterizes the congruence that is axiomatized by $\mathrm{CP}_{s}$.

Definition 6.13. Let $\sigma \in A^{u}$. The unary static evaluation function

$$
\text { sse }_{\sigma}: C_{\alpha(\sigma)} \rightarrow \mathcal{T}_{A}
$$

yields static evaluation trees and is defined as follows:

$$
\operatorname{sse}_{\sigma}(P)=m s e\left(\mathrm{\top} \triangleleft E^{\sigma} \triangleright P\right),
$$

where $E^{\sigma}$ is defined in Definition 6.5 and mse in Definition 5.12,

As an example, let $P=(a \triangleleft b \triangleright \mathrm{F}) \triangleleft a \triangleright \mathrm{T}$. We depict $s e(P)$ at the left-hand side. The static evaluation tree $\operatorname{sse}_{b a}(P)$ is depicted in the middle, and the static evaluation tree $s e_{a b}(P)$ is depicted at the right-hand side:
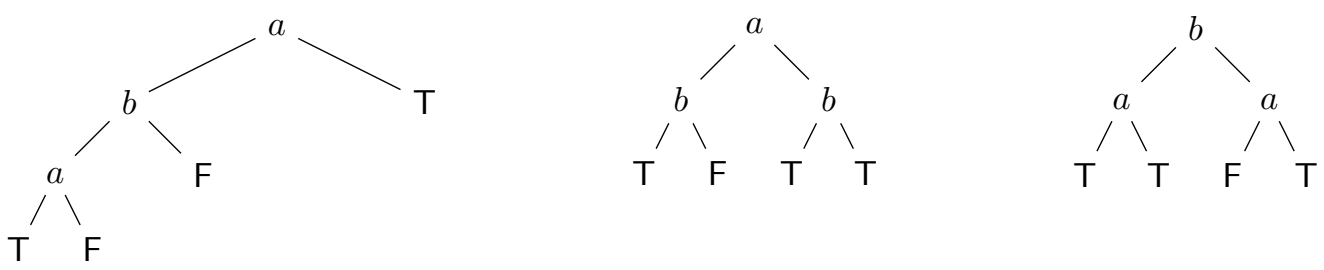

The two different static evaluation trees correspond to the different ways in which one can present truth tables for $P$, that is, the different possible orderings of the valuation values of the atoms occurring in $P$ :

\begin{tabular}{cc|c}
$a$ & $b$ & $(a \triangleleft b \triangleright \mathrm{F}) \triangleleft a \triangleright \mathrm{T}$ \\
\hline $\mathrm{T}$ & $\mathrm{T}$ & $\mathrm{T}$ \\
$\mathrm{T}$ & $\mathrm{F}$ & $\mathrm{F}$ \\
$\mathrm{F}$ & $\mathrm{T}$ & $\mathrm{T}$ \\
$\mathrm{F}$ & $\mathrm{F}$ & $\mathrm{T}$
\end{tabular}

\begin{tabular}{cc|c}
$b$ & $a$ & $(a \triangleleft b \triangleright \mathrm{F}) \triangleleft a \triangleright \mathrm{T}$ \\
\hline $\mathrm{T}$ & $\mathrm{T}$ & $\mathrm{T}$ \\
$\mathrm{T}$ & $\mathrm{F}$ & $\mathrm{T}$ \\
$\mathrm{F}$ & $\mathrm{T}$ & $\mathrm{F}$ \\
$\mathrm{F}$ & $\mathrm{F}$ & $\mathrm{T}$
\end{tabular}

The similarities between $s s e_{\sigma}$ and the function $s b f_{\sigma}$ can be exploited and lead to our final completeness result. 
Definition 6.14. Let $\sigma \in A^{u}$. Static valuation congruence over $\sigma$, notation $=_{s s e, \sigma}$, is defined on $C_{\alpha(\sigma)}$ as follows:

$$
P={ }_{s s e, \sigma} Q \Longleftrightarrow \operatorname{sse}_{\sigma}(P)=\operatorname{sse}_{\sigma}(Q) .
$$

The following characterization result immediately implies that for all $\sigma \in A^{u},{ }_{s s e, \sigma}$ is indeed a congruence relation on $C_{\alpha(\sigma)}$.

Proposition 6.15. Let $\sigma \in A^{u}$. For all $P, Q \in C_{\alpha(\sigma)}, P={ }_{s s e, \sigma} Q \Longleftrightarrow P={ }_{s b f, \sigma} Q$.

Proof. This follows by Proposition 5.13.

We end this section with a completeness result for static valuation congruence.

Theorem 6.16 (Completeness of $\left.\mathrm{CP}_{s}\right)$. Let $\sigma \in A^{u}$. For all $P, Q \in C_{\alpha(\sigma)}$,

$$
\mathrm{CP}_{s} \vdash P=Q \Longleftrightarrow P={ }_{s s e, \sigma} Q .
$$

Proof. Combine Theorem 6.12 and Proposition 6.15.

\section{Conclusions}

In 4 we introduced proposition algebra using Hoare's conditional $x \triangleleft y \triangleright z$ and the constants $\mathrm{T}$ and $\mathrm{F}$. We defined a number of varieties of so-called valuation algebras in order to capture different semantics for the evaluation of conditional statements, and provided axiomatizations for the resulting valuation congruences: $\mathrm{CP}$ (four axioms) characterizes the least identifying valuation congruence we consider, and the extension $\mathrm{CP}_{m e m}$ (one extra axiom) characterizes the most identifying valuation congruence below "sequential propositional logic" (SPL), while static valuation congruence, axiomatized by adding the simple axiom $\mathrm{F} \triangleleft x \triangleright \mathrm{F}=\mathrm{F}$ to $\mathrm{CP}_{m e m}$, can be seen as a characterization of SPL. In [3, 5] we introduced an alternative valuation semantics for proposition algebra in the form of Hoare-McCarthy algebras (HMA's) that is more elegant than the semantical framework provided in [4]: HMA-based semantics has the advantage that one can define a valuation congruence without first defining the valuation equivalence it is contained in.

In this paper, we use Staudt's evaluation trees 14 to define free valuation congruence as the relation $=_{s e}(\$ 2$, and this appears to be a relatively simple and stand-alone exercise, resulting in a semantics that is elegant and much simpler than HMA-based semantics $[3,5$, and the semantics defined in [4. By Theorem 2.11 ${ }_{s e}$ coincides with "free valuation congruence as defined in 4]" because both relations are axiomatized by CP (see [4, Thm.4.4 and Thm.6.2]). The advantage of "evaluation tree semantics" is that for a given conditional statement $P$, the evaluation tree $s e(P)$ determines all relevant evaluations, so $P={ }_{s e} Q$ is determined by evaluation trees that contain no more atoms than those that occur in $P$ and $Q$; this is comparable to how truth tables can be used in the setting of propositional logic.

In $₫ 3$ we define repetition-proof valuation congruence $={ }_{r p s e}$ on $C_{A}$ by $P={ }_{r p s e} Q$ if, and only if, $\operatorname{rpse}(P)=\operatorname{rpse}(Q)$, where $r p s e(P)=r p(s e(P))$ and $r p$ is a transformation function on evaluation trees. It is obvious that this transformation is "natural", given the axiom schemes (CPrp1) and CPrp2 that are characteristic for $\mathrm{CP}_{r p}(A)$. The equivalence on $C_{A}$ that we want to prove is

$$
\mathrm{CP}_{r p}(A) \vdash P=Q \Longleftrightarrow P={ }_{r p s e} Q
$$


by which $=_{r p s e}$ coincides with "repetition-proof valuation congruence as defined in [4]" because both are axiomatized by $\mathrm{CP}_{r p}(A)$ (see [4, Thm.6.3]). So, by equivalence (18), $=$ rpse is a congruence relation on $C_{A}$. However, we could not find a direct proof of this fact and we chose to simulate the transformation rpse by the transformation $r p b f$ on conditional statements, and to prove that the associated equivalence relation $=_{r p b f}$ is axiomatized by $\mathrm{CP}_{r p}(A)$, and is hence a congruence. This is Theorem 3.11, the proof of which depends on 4, Thm.6.35 and on Theorem 3.9, that is,

$$
\text { For all } P \in C_{A}, \mathrm{CP}_{r p}(A) \vdash P=\operatorname{rpbf}(P) \text {. }
$$

In order to prove (18) (which is Theorem 3.17), it is thus sufficient to prove that $=_{r p b f}$ and $={ }_{r p s e}$ coincide, and this is Proposition 3.16. Although it remains a challenge to find a direct and elegant proof of equivalence (18), we can conclude that repetition-proof evaluation trees and the valuation congruence $=_{r p s e}$ provide a full-fledged, simple and elegant semantics for $\mathrm{CP}_{r p}(A)$.

The structure of our completeness proofs of the axiomatizations for the other valuation congruences is very similar, although the case for static valuation congruence requires a slightly more complex proof (below we return to this point). Moreover, these axiomatizations are incremental: the axiom systems $\mathrm{CP}_{r p}(A)$ up to and including $\mathrm{CP}_{s}$ all share the axioms of $\mathrm{CP}$, and each succeeding system is defined by the addition of either one or two axioms, in most cases making previously added axiom(s) redundant. Given some $\sigma \in A^{u}$, this implies that in $C_{\alpha(\sigma)}$,

$$
{ }_{s e} \subseteq=_{r p s e} \subseteq=_{c s e} \subseteq=_{m s e} \subseteq=_{s s e, \sigma},
$$

where all these inclusions are proper. We conclude that for the valuation congruences $={ }_{s e}$ up to $={ }_{m s e}$, the associated evaluation trees provide a full-fledged, simple and elegant semantics.

The case for static valuation congruence over $C_{\alpha(\sigma)}$ for some $\sigma \in A^{u}$ is somewhat more involved. This semantics coincides with any standard semantics of propositional logic in the following sense:

$$
P={ }_{s s e, \sigma} Q \quad \text { if, and only if, } \quad \bar{P} \leftrightarrow \bar{Q} \text { is a tautology in propositional logic, }
$$

where $\bar{P}$ and $\bar{Q}$ refer to Hoare's definition [11]:

$$
\overline{x \triangleleft y \triangleright z}=(\bar{x} \wedge \bar{y}) \vee(\neg \bar{y} \wedge \bar{z}), \quad \bar{a}=a, \quad \overline{\mathrm{T}}=\mathrm{T}, \quad \overline{\mathrm{F}}=\mathrm{F} .
$$

Let $\sigma \in A^{u}$ and $a \in \alpha(\sigma)$. The fact that $=_{s s e, \sigma}$ identifies more than $={ }_{m s e}$ is immediately clear:

$$
\mathrm{F} \triangleleft a \triangleright \mathrm{F}={ }_{s s e, \sigma} \mathrm{F},
$$

while it is easy to see that $\mathrm{F} \triangleleft a \triangleright \mathrm{F} \neq \neq_{m s e} \mathrm{~F}$. Our proof that $\mathrm{CP}_{s}$ (and thus $\mathrm{CP}_{s t}$ ) is an axiomatization of static valuation congruence is slightly more complex than those for the other axiomatizations because in this case the evaluation of a conditional statement $P$ does not enforce a canonical order for the evaluation of its atoms, and therefore such an ordering should be fixed beforehand in order to construct an adequate evaluation tree. To this purpose, we can use any $\sigma \in A^{u}$ that covers the atoms in $P$.

A spin-off of our approach can be called "normalization functions for proposition algebra": for each valuation congruence $\mathrm{C}$ considered, two conditional statements are $\mathrm{C}$-valuation congruent if, and only if, the basic form function for $\mathrm{C}$ returns the same images 6

\footnotetext{
${ }^{5}$ This theorem requires that $|A|>1$, and so does the HMA approach in [3].

${ }^{6}$ We use the term "basic form" instead of "normal form" because according to CP, the obvious definition of a normal form $t \in C_{A}$ is that either $t \in A \cup\{\mathrm{T}, \mathrm{F}\}$, or $t$ satisfies the property "if $t_{1} \triangleleft t_{2} \triangleright t_{3}$ is a subterm of $t$, then $t_{2} \in A$ and it is not the case that $t_{1}=\mathrm{T}$ and $t_{3}=\mathrm{F}$ ". Also with respect to the other axiomatizations, a single atom $a \in A$ would be a typical normal form.
} 
We conclude with a brief digression on short-circuit logic, which we defined in [7] (see [5] for a quick introduction), and an example on the use of $\mathrm{CP}_{r p}(A)$. Familiar binary connectives that occur in the context of imperative programming and that prescribe short-circuit evaluation, such as \&\& (in C called "logical AND"), are often defined in the following way:

$$
P \& \& Q={ }_{\text {def }} \text { if } P \text { then } Q \text { else false, }
$$

independent of the precise syntax of $P$ and $Q$, hence, $P \& \& Q={ }_{\operatorname{def}} Q \triangleleft P \triangleright \mathrm{F}$. It easily follows that \&\& is associative (cf. Footnote 3). In a similarly way, negation can be defined by $\neg P={ }_{\operatorname{def}} \mathrm{F} \triangleleft P \triangleright \mathrm{T}$. In [7] we focus on this question:

Question 7.1. Which are the logical laws that characterize short-circuit evaluation of binary propositional connectives?

A first approach to this question is to adopt the conditional as an auxiliary operator, as is done in [7, 5, and to answer Question 7.1] using definitions of the binary propositional connectives as above and the axiomatization for the valuation congruence of interest in proposition algebra (or, if "mixed conditional statements" are at stake, axiomatizations for the appropriate valuations congruences). An alternative and more direct approach to Question 7.1 is to establish axiomatizations for short-circuited binary connectives in which the conditional is not used. For free valuation congruence, an equational axiomatization of short-circuited binary propositional connectives is provided by Staudt in [14, 13], where $\operatorname{se}(P \& \& Q)={ }_{\operatorname{def}} \operatorname{se}(P)[\mathrm{T} \mapsto \operatorname{se}(Q)]$ and $\operatorname{se}(\neg P)=_{\text {def }} \operatorname{se}(P)[\mathrm{T} \mapsto \mathrm{F}, \mathrm{F} \mapsto \mathrm{T}]$ (and where the function se is also defined for short-circuited disjunction), and the associated completeness proof is based on decomposition properties of such evaluation trees. For repetition-proof and contractive valuation congruence we conjecture that a finite equational axiomatization of the short-circuited binary propositional connectives does not exist if $|A|>2$. We conclude with an example on the use of $\mathrm{CP}_{r p}(A)$ that is based on [7, Ex.4].

Example 7.2. Let $A$ be a set of atoms of the form $\left(e==e^{\prime}\right)$ and $(\mathrm{n}=e)$ with $\mathrm{n}$ some initialized program variable and $e, e^{\prime}$ arithmetical expressions over the integers that may contain $\mathrm{n}$. Assume that $\left(e==e^{\prime}\right)$ evaluates to true if $e$ and $e^{\prime}$ represent the same value, and $(\mathrm{n}=e)$ always evaluates to true with the effect that $e$ 's value is assigned to $\mathrm{n}$. Then these atoms satisfy the axioms of $\mathrm{CP}_{r p}(A) 7$ Notice that if $\mathrm{n}$ has initial value 0 or $1,((\mathrm{n}=\mathrm{n}+1) \& \&(\mathrm{n}=\mathrm{n}+1)) \& \&(\mathrm{n}==2)$ and $(n=n+1) \& \&(n=2)$ evaluate to different results, so the atom $(n=n+1)$ does not satisfy the law $a$ \&\& $a=a$, by which this example is typical for the repetition-proof characteristic of $\operatorname{CP}_{r p}(A)$. End example.

We finally note that all valuation congruences considered in this paper can be used as a basis for systematic analysis of the kind of side effects that may occur upon the evaluation of short-circuited connectives as in Example 3.13, and we quote these words of Parnas [12:

"Most mainline methods disparage side effects as a bad programming practice. Yet even in well-structured, reliable software, many components do have side effects; side effects are very useful in practice. It is time to investigate methods that deal with side effects as the normal case."

\footnotetext{
${ }^{7}$ Of course, not all equations that are valid in the setting of Example 7.2 follow from $\mathrm{CP}_{r p}(A)$, e.g., $\mathrm{CP}_{r p}(A) \forall(0==0)=\mathrm{T}$. We note that a particular consequence of $\mathrm{CP}_{r p}(A)$ in the setting of short-circuit logic is $(\neg a$ \&\& $a$ ) \&\& $x=\neg a$ \&\& $a$ (cf. Example 3.13), and that Example 7.2 is related to the work of Wortel 15, where an instance of Propositional Dynamic Logic 8 is investigated in which assignments can be turned into tests; the assumption that such tests always evaluate to true is natural because the assumption that assignments always succeed is natural.
} 


\section{References}

[1] Bergstra, J.A., Bethke, I., and Rodenburg, P.H. (1995). A propositional logic with 4 values: true, false, divergent and meaningless. Journal of Applied Non-Classical Logics, 5(2):199-218.

[2] Bergstra, J.A. and Loots, M.E. (2002). Program algebra for sequential code. Journal of Logic and Algebraic Programming, 51(2):125-156.

[3] Bergstra, J.A. and Ponse, A. (2010). On Hoare-McCarthy algebras. Available at arXiv:1012.5059v1 [cs.LO].

[4] Bergstra, J.A. and Ponse, A. (2011). Proposition algebra. ACM Transactions on Computational Logic, Vol. 12, No. 3, Article 21 (36 pages).

[5] Bergstra, J.A. and Ponse, A. (2012). Proposition algebra and short-circuit logic. In Arbab, F. and Sirjani, M. (eds.), Proceedings of the 4th International Conference on Fundamentals of Software Engineering (FSEN 2011), Tehran. Volume 7141 of Lecture Notes in Computer Science, pages 15-31. Springer.

[6] Bergstra, J.A. and Ponse, A. (2015). Evaluation trees for proposition algebra: The case for free and repetition-proof valuation congruence. In: Meyer, R., Platzer, A., and Wehrheim, H. (eds.), Correct System Design (Olderog-Festschrift). Volume 9360 of Lecture Notes in Computer Science, pages 44-61, Springer.

[7] Bergstra, J.A., Ponse A., and Staudt, D.J.C. (2013). Short-circuit logic. Available at arXiv:1010.3674v4 [cs.LO,math.LO], 18 Oct 2010; this version (v4): 12 Mar 2013.

[8] Harel, D. (1984). Dynamic logic. In: Gabbay, D. and Günthner, F. (eds.), Handbook of Philosophical Logic, Volume II, pages 497-604. Reidel Publishing Company.

[9] Hayes, I.J., He Jifeng, Hoare, C.A.R., Morgan, C.C., Roscoe, A.W., Sanders, J.W., Sorensen, I.H., Spivey, J.M., and Sufrin B.A. (1987). Laws of programming. Communications of the $A C M, 3(8): 672-686$.

[10] Hoare, C.A.R. (1985). Communicating Sequential Processes. Prentice-Hall, Englewood Cliffs.

[11] Hoare, C.A.R. (1985). A couple of novelties in the propositional calculus. Zeitschrift für Mathematische Logik und Grundlagen der Mathematik, 31(2):173-178.

Republished in: Hoare, C.A.R. and Jones, C.B. (1989). Essays in Computing Science, pages 325-331. Prentice-Hall, Englewood Cliffs.

[12] Parnas, D.L. (2010). Really Rethinking 'Formal Methods'. Computer, 43(1):28-34, IEEE Computer Society (Jan. 2010).

[13] Ponse A. and Staudt, D.J.C. (2017). An independent axiomatization for free short-circuit logic. Available at arXiv:1707.05718v1 [cs.LO], 17 July 2017.

[14] Staudt, D.J.C. (2012). Completeness for two left-sequential logics. MSc. thesis Logic, University of Amsterdam (May 2012). Available at arXiv:1206.1936v1 [cs.LO].

[15] Wortel, L. (2011). Side effects in steering fragments. MSc. thesis Logic, University of Amsterdam (September 2011). Available at arXiv:1109.2222v1 [cs.LO]. 